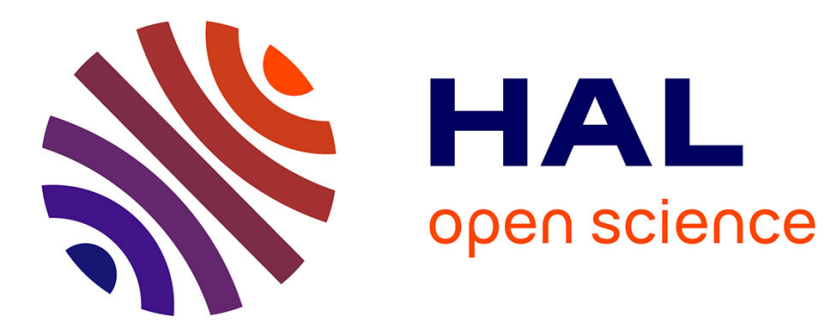

\title{
Kähler groups, R -trees, and holomorphic families of Riemann surfaces \\ Thomas Delzant
}

\section{To cite this version:}

Thomas Delzant. Kähler groups, R -trees, and holomorphic families of Riemann surfaces. Geometric And Functional Analysis, 2016, 10.1007/s00039-016-0355-x . hal-01261997

\section{HAL Id: hal-01261997 \\ https://hal.science/hal-01261997}

Submitted on 26 Jan 2016

HAL is a multi-disciplinary open access archive for the deposit and dissemination of scientific research documents, whether they are published or not. The documents may come from teaching and research institutions in France or abroad, or from public or private research centers.
L'archive ouverte pluridisciplinaire HAL, est destinée au dépôt et à la diffusion de documents scientifiques de niveau recherche, publiés ou non, émanant des établissements d'enseignement et de recherche français ou étrangers, des laboratoires publics ou privés. 


\title{
Kähler groups, r -trees, and holomorphic families of Riemann surfaces.
}

\author{
Thomas Delzant \\ IRMA, Université de Strasbourg et CNRS
}

January 26, 2016

\begin{abstract}
Let $X$ be a compact Kähler manifold, and $g$ a fixed genus. Due to the work of Parshin and Arakelov, it is known that there are only a finite number of non isotrivial holomorphic families of Riemann surfaces of genus $g \geqslant 2$ over $X$. We prove that this number only depends on the fundamental group of $X$. Our approach uses geometric group theory (limit groups, $\mathrm{R}$-trees, the asymptotic geometry of the mapping class group), and Gromov-Shoen theory. We prove that in many important cases limit groups (in the sense of Sela) associated to infinite sequences of actions of a Kähler group on a Gromov-hyperbolic space are surface groups and we apply this result to monodromy groups acting on complexes of curves.
\end{abstract}

\section{Introduction}

Let $X$ be a compact, connected Kähler manifold. A holomorphic family of Riemann surfaces of genus $g$ over $X$ is a pair $(Y, \pi)$ where $Y$ is a complex manifold and $\pi: Y \rightarrow X$ a holomorphic submersion whose fibers are Riemann surfaces of genus $g$ (we assume $g \geqslant 2$ ). It is called non isotrivial if the family of Riemann surfaces $Y_{s}=\pi^{-1}(s)$ is not constant in the moduli space of Riemann surfaces. A holomorphic family of Riemann surfaces determines a monodromy, which is a homomorphism $\varphi$ from the fundamental group $\pi_{1}\left(X, s_{0}\right)$ to the mapping class group $M(S)$ of the topological surface underlying $Y_{s_{0}}$, the fiber at the point $s_{0}$.

A fundamental result due to Parshin and Arakelov ([Ar], [Par]) and answering a question of Shafarevich asserts that given a Riemann surface $B$ the set of families of given genus over $B$ is finite. Another proof based on the study of the action of the monodromy on the Teichmüller spaces, as been given by Imayoshi and Shiga [Im-Sh], see also the article of McMullen [McM].

Another consequence of the study of $[\mathrm{Ar}]$ is that the number of non isotrivial families over a projective manifold $X$ can be bounded in terms of this manifold. A uniform result has even been described by L. Caporaso [Ca] who proved that 
the Hilbert polynomial of a complex surface which is a non singular bundle of genus $g \geqslant 2$ over a base of genus $p \geqslant 2$ can only take a finite number of values. In particular, given a topological surface $\Sigma_{p}$ of genus $p$ and a topological surface $S$ (of genus $g$ ), the cardinality of the set of homomorphisms from the fundamental group of $\Sigma_{p}$ to the mapping class group of $S$ which can be realized as a monodromy (for a certain complex structure on $\Sigma$ ) is finite up to conjugacy at the target and automorphism at the source.

In this article, we want to give a bound of the number of families over a base $X$ in terms of its fundamental group $\Gamma=\pi_{1}(X)$, independent of the manifold $X$. Before stating our main result let us recall some definitions.

A finitely presented group $\Gamma$ is Kähler if it can be realized as the fundamental group of a compact Kähler manifold. The group $\Gamma$ fibers if there exist a topological 2-orbifold $\Sigma$ of hyperbolic type together with a surjective homomorphism $\pi: \Gamma \rightarrow \pi_{1}^{\text {orb }}(\Sigma)$ whose kernel is finitely generated. This is equivalent to the fact that every compact Kähler manifold $X$ with fundamental group $\Gamma$ admits a holomorphic map with connected fibers on a complex hyperbolic 1-orbifold whose underlying topological orbifold is $\Sigma$ (see paragraph 2.1). Analogously, one says that the family $Y$ over $X$ factors through a curve $B$ if there exist a Riemann orbifold $B$ and a holomorphic map $q: X \rightarrow B$ so that $Y$ is the pullback of a family over $B$. We shall see (see Corollary 1 in paragraph 5.3 ) that this property only depends on the monodromy of the family and not on the manifold $X$. We will prove (see Theorem 9 in 5.4) :

Theorem 1 Let $\Gamma$ be a Kähler group and $S$ a topological surface. There exists only finitely many conjugacy classes of homomorphisms $\varphi: \Gamma \rightarrow M(S)$ which can be realized as the monodromy of a holomorphic family of Riemann surfaces on some Kähler manifold with fundamental group $\Gamma$, but do not factor through a curve.

Combining this result with the case of curves ([Ca]) one obtains that the number of non isotrivial families over a Kähler manifold $X$ can be bounded in terms of its fundamental group (see Corollary 2 in Paragraph 5.4).

In fact, Theorem 1 appears as a very special case of a general factorization Theorem for actions of Kähler groups on Gromov-hyperbolic spaces (see Theorem 6 in Paragraph 3.4).

Let $H$ be a hyperbolic space in the sense of Gromov ([Gr]), and $G$ a subgroup of the group of isometries of $H$. If $\Gamma$ is a finitely generated group, one can study infinite sequences of non elementary homomorphisms from $\Gamma$ to $G$ with an asymptotic method (sometimes called the Bestvina-Paulin method). Let $\Sigma$ be a fixed set of generators of $\Gamma$. The energy of the homomorphism $\varphi$ is $e(\varphi)=\operatorname{Min}_{x \in H} \operatorname{Max}_{s \in \Sigma} d(x, \varphi(s) x)$. An infinite sequence of pairwise non conjugate homomorphisms is diverging if $\lim \sup e\left(\varphi_{n}\right)=+\infty$. After choosing some ultrafilter, infinite sequences of diverging energy converge to an action of $\Gamma$ on some R-tree : the asymptotic cone of $H$ (see Paragraph 3.2). Due to 
the fundamental work of Gromov-Shoen [Gr-Sh], one deduces that the Kähler group "fibers", i.e. admits an epimorphism $\pi$ to a 2-orbifold group with finitely generated kernel $K$.

In order to prove that for some (and in fact almost every) integer $n, \varphi_{n}$ factor through this orbifold group, one need to check that this normal subgroup $K$ belongs to the kernel of $\varphi_{n}$. This is why we introduce the property of weak acylindricity for an action of a group on a hyperbolic space, which enable to prove that if the limit action is not elementary, for some integer $n, \varphi_{n}(K)$ is an elliptic subgroup of $G$, normal in $\varphi_{n}(\Gamma)$. In many cases, a normal subgroup of a group acting effectively on a hyperbolic space cannot be elliptic, and this is enough to insure that $K$ is in the kernel of $\varphi_{n}$.

Unfortunately this natural method cannot be applied directly to the mapping class group acting on the complex of curves (which is a hyperbolic space due to the work of Masur and Minsky [Ma-Mi]) as there is no reason that an infinite sequence of homomorphisms to the mapping class group acting on the complex of curves has diverging energy. The whole machinery of Bestvina, Bromberg, and Fujiwara [Be-Br-Fu] which creates a proper action of the mapping class group on a finite product of hyperbolic spaces will be needed together with the work of Berhstock, Drutu and Sapir [Be-Dr-Sa] on the asymptotic geometry of this group.

In the first paragraph we review some basic results concerning Kähler groups acting on R-trees, in the second paragraph we recall the Bestvina-Paulin method to construct actions on R-trees and we introduce the notion of a limit group associated to an infinite sequence. This theory is applied to the study of Kähler groups, and in particular we prove that in many cases, limit groups of Kähler groups are surface groups. In the last paragraph these results are combined with the description of the asymptotic geometry of the mapping class group to study monodromies.

Acknowledgments. I would like to thank Pierre Py for many comments and remarks on this subject, and Chloé Perin for discussions concerning limit groups, and especially the referee for several important comments, questions and suggestions.

\section{Kähler groups and R-trees}

\subsection{2 -Orbifolds and their fundamental groups}

Let us recall some basic facts about 2 - orbifolds, their fundamental groups and geometric structures as introduced by W. Thurston ([Th] Chapter 13).

An (oriented) 2-orbifold is a topological (oriented) compact surface $S$ endowed with a finite set of marked points $\left\{\left(p_{1}, m_{1}\right), \ldots,\left(p_{k}, m_{k}\right)\right\}$, where $m_{i}$ is an integer greater than 2 . We shall denote it by $\Sigma=\left\{S ;\left(p_{1}, m_{1}\right), \ldots,\left(p_{k}, m_{k}\right)\right\}$. For each point $p_{i}$, let $\gamma_{i}$ be a small simple loop which is the boundary of a small 
embedded disc $D_{i}$ centered at $p_{i}$ and chosen so that the disks $\left(D_{i}\right)_{1 \leqslant i \leqslant k}$ are disjoints.

The fundamental group of $\Sigma$ is defined as a quotient :

$$
\pi_{1}^{\text {orb }}=\pi_{1}\left(S \backslash\left\{p_{1}, \ldots, p_{k}\right\}\right) / \ll \gamma_{1}^{m_{1}}, \ldots, \gamma_{k}^{m_{k}} \gg .
$$

The Euler characteristic of $\Sigma$ is $\chi(\Sigma)=2-2 g-k+\sum_{1 \leqslant i \leqslant k} \frac{1}{m_{i}}$. A 2-orbifold is called of hyperbolic type if its Euler characteristic $\chi(X)$ is strictly negative.

If $\Lambda$ is a co-compact lattice in $\operatorname{PSL}(2, \mathrm{R})$, the quotient of the unit disk $D$ by $\Lambda$ has naturally a structure of a 2-orbifold : $D / \Lambda$ is a topological oriented surface $S$ and modulo $\Lambda$, only finitely many points $\left(p_{i}\right)_{1 \leqslant i \leqslant k}$ have a non trivial isotropy group, which is a finite cyclic subgroup of order $\left(m_{i}\right)_{1 \leqslant i \leqslant k}$ of $\Lambda$. If $\Sigma=\left\{S ;\left(p_{i}, m_{i}\right)\right\}$ is the underlying orbifold, one proves that $\Lambda=\pi_{1}^{\text {orb }}(\Sigma)$. A hyperbolic structure on $\Sigma$ is a realization of its fundamental group as a cocompact lattice in $\operatorname{PSL}(2, \mathrm{R})=\operatorname{Aut}(D)$, the automorphism group of the unit disc in $\mathrm{C}$

A complex structure on $\Sigma$ is a complex structure on $S$, marked at the points $p_{i}$, and the uniformization Theorem for 2-orbifolds implies that there is a oneto-one correspondence between hyperbolic and complex structures on general 2-orbifolds.

Let us denote $Y$ the orbifold $\Sigma=\left\{S ;\left(p_{1}, m_{1}\right), . .\left(p_{k}, m_{k}\right)\right\}$ endowed with a complex structure. In order to define holomorphic maps with values in $Y$, let us choose for every $i$ a small closed disk $D_{i}$ around $p_{i}$. Let $G_{i}:\left(\tilde{D}^{m_{i}}, p_{i}\right)$ $\left(D_{i}, p_{i}\right)$ be the $m_{i}$ - th cover ramified at the origin. If $X$ is a complex manifold, a map $f: X \rightarrow \Sigma$ is called holomorphic if it is holomorphic in the usual sense in $X-f^{-1}\left\{\left(p_{i}\right)_{1 \leqslant i \leqslant n}\right\}$, and if for every $i$ and every point $x$ such that $f(x)=p_{i}$, the map $f$ admits a local holomorphic lift through the map $G_{i}$. In other words, $f-p_{i}$ is locally the $m_{i}-$ th power of a holomorphic map. This definition enables us to endow $Y$ with the Kobayashi metric which coincides with the hyperbolic structure given by the uniformization Theorem.

The following result is due to F. Catanese ([Cat] Theorem 5.14). We propose below a different proof based on the work of J. Carleson and D. Toledo [Ca-To].

Theorem 2 Let $\Gamma$ be the fundamental group of a compact Kähler manifold $X$ and $\Lambda$ the fundamental group of a compact 2-orbifold $\Sigma$ of hyperbolic type. The following are equivalent:

i. There exists a surjective homomorphism $\psi: \Gamma \rightarrow \Lambda$ with finitely generated kernel.

ii. There exists a complex structure $Y$ on $\Sigma$ together with a holomorphic map $X \rightarrow Y$ with connected fibers.

Proof Let us choose some discrete co-compact action of $\Lambda$ on the unit disk, with orbifold quotient $Y_{\text {aux }}$ (an auxiliary hyperbolic/complex structure on $\Sigma$ ). 
The theory of harmonic maps (developed by J. Carlson and D. Toledo [Ca-To]) proves that there exists an equivariant harmonic map from the universal cover of $X$ to $D$ which leads to a differentiable map $f$ from $X$ to $Y_{\text {aux }}$. Using a Bochner formula, [Ca-To] prove that the map $f$ is pluriharmonic and that the connected components of the fibers of $f$ are complex hypersurfaces. The set of connected components of fibers of $f$ is a complex one dimensional orbifold $Z$ , i.e a Riemann surface with a finite set of marked points, the multiple fibers with their multiplicity. Let $\Sigma^{\prime}$ be the topological orbifold underlying $Z$. There exists a continuous map $q: \Sigma^{\prime} \rightarrow \Sigma$ that induces a surjective homomorphism on (orbifolds) fundamental groups. In order to prove that this homomorphism is an isomorphism, we adapt the argument of F. Catanese. Let us consider the image of $\operatorname{ker}(\psi)$ in $\pi_{1}^{\text {orb }}\left(\Sigma^{\prime}\right)$. As $\operatorname{ker}(\psi)$ is finitely generated and $\psi^{\prime}: \Gamma \rightarrow \pi_{1}^{\text {orb }}\left(\Sigma^{\prime}\right)$ is onto, the image of $\operatorname{ker}(\psi)$ in $\pi_{1}^{\text {orb }}\left(\Sigma^{\prime}\right)$ must be trivial or have finite index (finitely generated normal subgroups in 2 -orbifold groups have finite index). This group, which is the kernel of $q_{*}$, cannot have finite index as $q_{*}: \pi_{1}^{\text {orb }}\left(\Sigma^{\prime}\right) \rightarrow \pi_{1}^{\text {orb }}(\Sigma)$ is onto, thus it is trivial and $q$ is an isomorphism.

Using the fact that holomorphic maps are 1-Lipshitz for the Kobayashi metric (which is the hyperbolic metric on a hyperbolic 2-orbifold), one proves the following Proposition (see [De] Theorem 2 or [Co-Si] for a purely algebraic proof).

Proposition 1 Let $X$ be a compact complex manifold. There exist only finitely many pairs $\left(Y_{i}, F_{i}\right)$ where $Y_{i}$ is a hyperbolic/complex 2-orbifold and $F_{i}$ a holomorphic map from $X$ to $Y_{i}$.

This suggests the following definition (see [ABCKT] Chapter 2, Paragraph $3)$.

Definition 1 A Kähler group $\Gamma$ fibers if there exists a 2-orbifold of hyperbolic type $\Sigma$ and a surjective homomorphism $\Gamma \rightarrow \pi_{1}^{\text {orb }}(\Sigma)$ whose kernel is finitely generated.

We wish to emphasize that this definition only depends on $\Gamma$ and not on the choice of a Kähler manifold with $\Gamma$ as a fundamental group. Combining the previous two results, one gets the following :

Proposition 2 Let $\Gamma$ be a Kähler group. There exists a finite family of pairs $\left(\Sigma_{i}, \pi_{i}\right)_{1 \leqslant i \leqslant p}$ of 2-orbifolds of hyperbolic type and surjective homomorphisms $\pi_{i}: \Gamma \rightarrow \pi_{1}^{\text {orb }}\left(\Sigma_{i}\right)$ with finitely generated kernel, such that for every Kähler manifold $X$ with fundamental group $\Gamma$, every one dimensional hyperbolic orbifold $Y$ and every holomorphic map $F: X \rightarrow Y$ with connected fibers, there exists an integer $i$ such that the topological orbifold underlying $Y$ is isomorphic to $\Sigma_{i}$ by an isomorphism inducing $\pi_{i}$ on the fundamental groups.

\section{$2.2 \quad$ Trees}

Recall that an $\mathrm{R}$-tree is a connected geodesic metric space which is 0 -hyperbolic (see $[\mathrm{Be} 2]$ or $[\mathrm{Ka}]$ for an introduction to this subject). 
An $\mathrm{R}$-tree, endowed with an action of a group $\Gamma$ is called minimal if it does not contain an invariant subtree. If the group $\Gamma$ is finitely generated, such a minimal subtree exists and is unique. The action is called non-elementary if it is neither elliptic (fixing a point) nor axial (fixing a line but no point on this line) nor parabolic (fixing a unique point at infinity).

The main example of a Kähler group acting on an $\mathrm{R}$ - tree is the fundamental group of a Riemann surface (or 2-orbifold) endowed with a holomorphic quadratic differential $\omega$ : the $\mathrm{R}$ - tree is the set of leaves of the real part of $\omega$.

After the fundamental work of Gromov-Schoen [Gr-Sh] several authors ([Ko$\mathrm{Sh}],[\mathrm{Su}])$ studied actions of Kähler groups on $\mathrm{R}$-trees. The following Theorem summarizes the situation.

Theorem 3 Let $\Gamma$ be a Kähler group acting on an $\mathrm{R}$-tree $T$. Assume that $T$ is minimal and is not a line. Then $\Gamma$ fibers. More precisely, there exist a 2orbifold $\Sigma$ of hyperbolic type, a surjective map $\pi: \Gamma \rightarrow \pi_{1}^{\text {orb }}(\Sigma)$ with finitely generated kernel, an action of the fundamental group of $\Sigma$ on an $\mathrm{R}-$ tree $T^{\prime}$, and a $\pi$-equivariant map $T^{\prime} \rightarrow T$. If the action of $\Gamma$ on $T$ is faithful, then $\pi$ is an isomorphism.

If $T$ is a simplicial locally finite tree, the proof is explained in [Gr-Sh] ; the general case is sketched in Paragraph 9.1 of the same article. Let us recall the main steps of the proof.

Let $X$ be a compact Kähler manifold with fundamental group $\Gamma$. One constructs a $\Gamma$-equivariant harmonic map $h$ from the universal cover of $X$ to the tree $T$ with finite $\Gamma$-energy ([Gr-Sh], [Ko-Sh]). The regularity of this harmonic map ([Gr-Sh]) has been detailed by [Su]; in particular, its set of singular points $X_{\text {sing }}$ has codimension 2 and the image of a connected fundamental domain in $\tilde{X}$ appears to be a finite tree (the convex hull of a finite number of points). At this point one can copy the argument of [Gr-Sh]: using the Kähler structure, one proves that the map $h$ is pluriharmonic: outside from $X_{\text {sing }}$ it is locally the real part of a holomorphic function, and there exists a holomorphic quadratic differential $\omega$ on $X$ such that locally $\pm d h=\omega$. Using the fact that the tree is not a line one proves that one leaf of the foliation defined by $\omega$ on $X$ is singular (entirely contained in the set $\omega=0$ ) hence compact. One deduces that all leaves are compact and $X$ fibers on some hyperbolic 2-orbifold $\Sigma$ with fibers the leaves of $\omega$. Thus $\omega$ comes from $\Sigma$ and if $T_{\omega}$ stands for the $\mathrm{R}-$ tree dual to the leaves of $\omega$, the harmonic map $h$ factors through the $\pi_{1}(\Sigma)$-equivariant map from $D$ to $T_{w}$.

The case of axial actions of Kähler groups is also quite useful (see [De2]).

Theorem 4 Let $\Gamma$ be a Kähler group with an isometric action on a line (isometric to R). If the kernel of this action is not finitely generated, the group $\Gamma$ fibers; there exists a 2-orbifold of hyperbolic type and a surjective morphism with finitely generated kernel $\pi: \Gamma \rightarrow \pi_{1}^{\text {orb }}(\Sigma)$ such that the action factors through $\pi$. 
Proof Let $X$ be a compact manifold with fundamental group $\Gamma$. In the case of an oriented line, such an action is defined by the real part of an holomorphic form and [De2] applies. In the general case, the action defines a holomorphic quadratic differential $\omega$ which becomes a differential on a (perhaps) ramified double cover. All the leaves of this form are compact, hence all the leaves of $\omega$ are compact and the same argument as in Theorem 3 completes the proof.

\section{$3 \quad$ Limit groups and $\mathrm{R}$-trees as limit spaces}

In many cases, actions of groups on R-trees are obtained by a limit process of actions on hyperbolic spaces, as observed by Bestvina [Be1] and Paulin $[\mathrm{Pa}]$.

\subsection{Limit spaces and limit groups.}

Let $\Gamma$ be a finitely generated group, $\left(H_{n}, x_{n}, d_{n}\right)$ a sequence of pointed metric spaces, and $\varphi_{n}: \Gamma \rightarrow \operatorname{Isom}\left(H_{n}\right)$ a sequence of isometric actions of $\Gamma$ on $H_{n}$. Let us recall the definition of the $\omega$ - limit (or asymptotic cone) associated to such a sequence (see [B-H], pp. 77-80).

In order to study the asymptotic behavior of this sequence of actions, let us fix a non principal ultrafilter $\omega$ on N. Recall (see $[\mathrm{BH}] \mathrm{p} .78$ ), that $\omega$ is a subset of the set of infinite subsets of $\mathrm{N}$ such that, if $A, B \in \omega$ then $A \cap B \in \omega$, the complementary $F^{c}$ of every finite set $F$ is in $\omega$, and $\omega$ is maximal for these properties. One says that a family of Propositions $\left(P_{n}\right)_{n \in \mathrm{N}}$ is true $\omega$-almost surely if the subset of $\mathrm{N}$ such that $P_{n}$ is true belongs to $\omega$. If $\left(u_{n}\right)_{n \in \mathrm{N}}$ is a sequence in a Hausdorff topological space, one says that $l$ is an $\omega$-limit of $\left(u_{n}\right)$ and writes $l=\lim _{\omega} u_{n}$, if for every neighborhood $U$ of $l$, the set $\left\{n \in \mathrm{N} / u_{n} \in U\right\}$ is an element of $\omega$. In a compact Hausdorff space every sequence has a unique $\omega$-limit. In particular every bounded sequence of real numbers has a $\omega$-limit in $\mathrm{R}$ and every sequence of positive numbers has a $\omega$-limit in $\mathrm{R} \cup\{+\infty\}$. The following definitions are useful to describe the asymptotic behavior of a family of actions.

Definition 2 The space $\Pi^{\text {bounded }} H_{n}$ is the subset of the usual product $\Pi_{n \in \mathrm{N}} H_{n}$ made with sequences $\left(y_{n}\right)_{n \in \mathrm{N}}$ such that $\lim _{\omega} d\left(x_{n}, y_{n}\right)<\infty$.

The limit space $H_{\omega}=\lim _{\omega}\left(H_{n}, d_{n}, x_{n}\right)$ is the quotient of the set $\Pi^{\text {bounded }} H_{n}$ by the equivalence relation $\lim _{\omega} d_{n}\left(y_{n}, z_{n}\right)=0$.

This limit space $\lim _{\omega}\left(H_{n}, d_{n}, x_{n}\right)=H_{\omega}$ has a natural base-point namely the equivalence class of $\left(x_{n}\right)$ and a natural distance $d_{\omega}$ defined by $d_{\omega}(y, z)=$ $\lim _{\omega} d\left(y_{n}, z_{n}\right)$. It is a complete metric space. If for every element $g$ in a generating set of $\Gamma$, the sequence $d_{n}\left(x_{n}, \varphi_{n}(g) x_{n}\right)$ is bounded, the group $\Gamma$ acts isometrically on $H_{\omega}$, as $\Gamma$ acts on $\Pi^{\text {bounded }} H_{n}$.

Definition 3 The stable kernel of $\left(\varphi_{n}\right)_{n \in \mathrm{N}}$ is the normal subgroup $N_{\omega}$ of $\Gamma$ defined as $N_{\omega}=\left\{g \in \Gamma /\left\{n / \varphi_{n}(g)=e\right\} \in \omega\right\}$. 
Definition 4 The limit group $\Gamma_{\omega}$ is the quotient $\Gamma / N_{\omega}$.

By definition, the limit group $\Gamma_{\omega}$ acts on the limit space $H_{\omega}$. The following Proposition emphasizes the importance of finiteness properties of kernels of actions.

Proposition 3 Let $K$ be a finitely generated subgroup contained in $N_{\omega}$. Then for $\omega$-almost every integer $n, K \subset \operatorname{Ker}\left(\varphi_{n}\right)$ the kernel of the action of $\varphi_{n}$. For $\omega$-almost all $n, \varphi_{n}$ factors through $\Gamma / K$.

Proof In fact, if $\sigma=\left\{a_{1}, \ldots, a_{n}\right\}$ is a finite set of generators of $K$, for every $i$ the set $A_{i}=\left\{n \in \mathrm{N} / \varphi_{n}\left(a_{i}\right)=e\right\}$ is an element of $\omega$. The finite intersection $\cap A_{i}$ is therefore an element of $\omega$.

\subsection{Hyperbolic metric spaces and R-trees}

Let $H$ denotes a $\delta$-hyperbolic space (in the sense of Gromov [Gr]), $\Gamma$ a finitely generated group, $\sigma$ be a fixed set of generators of $\Gamma$, and $\varphi: \Gamma \rightarrow \operatorname{Isom}(H)$ an isometric action.

The energy function of the action is the function defined by $e(x)=\operatorname{Sup}_{s \in \sigma} d(\varphi(s) . x, x)$, and the energy of the action is the minimum $e(\varphi)=\operatorname{Min}_{x \in H} e(x)$. If the action of $\Gamma$ on $H$ is not elementary (neither elliptic, nor parabolic, nor loxodromic), then the function $e(x)$ is metrically proper (it goes to infinity with the distance of $x$ to a fixed base-point). In this case, the set $\{x \in H / e(x) \leqslant e(\varphi)+1\}$ is not empty and has bounded diameter. If $H$ is furthermore a $\operatorname{CAT}(-1)$ complete metric space, the minimum is in fact achieved, as $e$ is a convex (along geodesics) metrically proper function. (See $[\mathrm{Be} 2]$ ).

The main result concerning (ultra)-limits of actions of a group acting on a hyperbolic space is due to M. Bestvina and F. Paulin see ([Be1], [Be2],[Br-Ha], [Ka], [Ko-Sh], [Pa]).

Theorem 5 Let $\Gamma$ be a finitely generated group, $(H, d)$ a hyperbolic space and $\varphi_{n}: \Gamma \rightarrow \operatorname{Isom}(H)$ be a sequence of actions. Let $x_{n}$ be a sequence of base points with $e_{n}\left(x_{n}\right) \rightarrow \infty$, and $d_{n}$ be the renormalized distance: $d_{n}=\frac{d}{e_{n}\left(x_{n}\right)}$. Then the limit space $\lim _{\omega}\left(H, d_{n}, x_{n}\right)$ is a complete $\mathrm{R}$-tree $H_{\omega}$.

Proof (Compare [Be2], [Ko-Sh], [Gr2] 2.B.b). Recall (see [Co-De-Pa] for a detailed presentation of the subject) that a complete metric space $H$ with a base point $x$ is hyperbolic if :

$$
\forall y, z, t \in H, \quad<y, z>\geqslant \min (<y, t>;<t, z>)-\delta,
$$

where $\langle y, z>=1 / 2(d(x, y)+d(x, z)-d(y, z))$.

Recall that such a space is geodesic if :

$$
\forall y, z \in H \quad \exists m \in H / d(y, m)=d(m, z)=1 / 2 d(y, z) .
$$


These two properties (geodesicity, hyperbolicity) are formulated in the first order language of metric spaces : a finite number of quantifiers and inequalities only involving distances. Therefore, they behave nicely through ultra-limits. Let $\delta_{n}=\frac{\delta}{e_{n}}$ the hyperbolicity constant of $\left(H, d_{n}\right)$. As $\lim _{\omega} \delta_{n}=0$, the limit space $H_{\omega}$ of the family $\left(H, d_{n}\right)$ of $\delta_{n}$ hyperbolic geodesic spaces is a 0 -hyperbolic geodesic space, hence an R-tree. Using the finiteness of the generating set $\sigma$, one proves that $e\left(x_{\omega}\right)=1$.

Changing base points. Let $\Gamma$ be a f.g. group, and $\varphi_{n}$ a sequence of actions in some hyperbolic space $X, e_{n}(x)$ the energy function of the action $\varphi_{n}$, $e_{n}=\operatorname{Inf}_{x}\left(e_{n}(x)\right)$ and $x_{n}$ a sequence of point in $X$. Assume that $\lim _{\omega} e_{n}\left(x_{n}\right)=$ $+\infty$. Then $\Gamma$ acts on the limit tree $T=\lim _{\omega}\left(X_{n}, x_{n}, d / e_{n}\left(x_{n}\right)\right)$. The next proposition shows that if the limit space is not trivial, it does not really depend on the choice of the sequence. However, in general, it depends on the choice of the ultra-filter.

Proposition 4 If the limit action of $\Gamma$ on $T=\lim \left(X, \frac{d}{e_{n}\left(x_{n}\right)}, x_{n}\right)$ is not elliptic, then $\lim _{\omega} e_{n}=\infty$. If furthermore it is non elementary, then for some $D$ and omega-almost integer, $e_{n}\left(x_{n}\right) \leq(D+1) e_{n}$. Up to a scaling factor, the limit tree does not depends on the choice of the sequence $\left(x_{n}\right)$.

Proof Assume firstly that the limit action of $\Gamma$ on this limit tree is not elliptic, and let $y_{\omega} \in T_{\omega}$ be such that $e_{\omega}(y)$ is minimal for $y=y_{\omega}$. Let $y=\lim _{\omega}\left(y_{n}\right)$. As $e_{\omega}(y)=\lim _{\omega} \frac{e_{n}\left(y_{n}\right)}{e_{n}\left(x_{n}\right)}$, have $e_{n}\left(y_{n}\right)=e_{n}\left(x_{n}\right) e_{\omega}+o\left(e_{n}\left(x_{n}\right)\right)$. In particular the limit $\lim _{\omega}\left(X, y_{n}, d / e_{n}\left(y_{n}\right)\right)$ is the same tree but with the distance $d_{\omega}^{\prime}=\frac{d_{\omega}}{e_{\omega}}$.

Assume now that the action of $\Gamma$ on the limit tree is not elementary.

The set $y \in T_{\omega}, y=\lim _{\omega}\left(y_{n}\right)$ be such that $e_{\omega}(y)$ is minimal is bounded of diameter $D$ (if not $\Gamma$ would fix a point in the boundary of this tree). Let $z_{n} \in X$ with $e_{n}\left(z_{n}\right) \leq e_{n}(y)+o\left(e_{n}\left(x_{n}\right)\right)$. Let us prove that $\lim _{\omega} \frac{d_{n}\left(y_{n}, z_{n}\right)}{e_{n}\left(y_{n}\right)}<\infty$.

If not, for $\omega$ almost all $n$, we have : $\frac{d_{n}\left(y_{n}, z_{n}\right)}{e_{n}\left(y_{n}\right)}>D+1$. Let $z_{n}^{\prime} \in\left[y_{n}, z_{n}\right]$ be a point at a distance $e_{n}\left(y_{n}\right) \cdot(D+1)$. Recall that the distance function in hyperbolic space is $8 \delta$-convex : if $c_{1}(t), c_{2}(t)$ are two geodesics parametrized by arc length on the segment $[0, L], d\left(c_{1}(t . L), c_{2}(t . L)\right) \leq(1-t) d\left(c_{1}(0), c_{2}(0)\right)+$ $t d\left(c_{1}(L), c_{2}(L)+8 \delta\right.$ (see [Co-De-Pa], Corollary 5.3, page 117). Therefore, $e_{n}\left(z_{n}^{\prime}\right) \leq$ $\max \left(e_{n}\left(z_{n}\right), e_{n}\left(y_{n}\right)\right)+8 \delta$. The point $z^{\prime}=\lim _{\omega}\left(z_{n}^{\prime}\right)$ is a point at a distance $D+1$ of $y$ with $e\left(z^{\prime}\right) \leq e(y)$, contradiction.

Assume that the action of $\Gamma$ on the limit tree is not elliptic.

As $\Gamma$ is finitely generated, there exists a $g \in \Gamma$ which is an hyperbolic isometry of $T_{\omega}$, of translation length $[g]_{\omega}>0$. Let $d_{\varphi_{n}(g)}(x)=d\left(\varphi_{n}(g) x, x\right)$ the displacement function of $\varphi_{n}(g),\left[\varphi_{n}(g)\right]$ its minimum, and $C_{n}=\left\{x / d\left(x, \varphi_{n}(g) x\right) \leq\right.$ $\left.\max \left(\left[\varphi_{n}(g)\right], 20 \delta\right)\right\}$ the set where it is minimal. We know (see for instance [De-Gr], prop. 2.3.3 (1)) that $\left.2 d\left(x_{n}, C_{n}\right) \leq d\left(\varphi_{n}(g) x_{n}, x_{n}\right)\right)-\left[\varphi_{n}(g)\right]+8 \delta \leq$ $k e_{n}\left(x_{n}\right)+8 \delta$, if $k$ denote the word length $g$. Therefore, if $p_{n}$ is a point where 
$d\left(x_{n}, C_{n}\right)$ is minimal, $\frac{d\left(x_{n}, p_{n}\right)}{e_{n}\left(x_{n}\right)}$ is bounded, and $p_{n}$ converges to a point in the ultra-limit. Then for every $\left.x, k \cdot e_{n} \geq d\left(\varphi_{n}(g) x\right), x\right) \geq d\left(\varphi_{n}(g)\left(p_{n}\right), p_{n}\right) \geq$ $[g]_{\omega} e_{n}\left(x_{n}\right)+o\left(e_{n}\left(x_{n}\right)\right.$, and $\frac{e_{n}}{e_{n}\left(x_{n}\right)} \geq 1 / k[g]_{\omega}+o(1)$.

\subsection{Acylindricity, WWPD, weak acylindricity.}

In this subsection we study the notion of weak acylindricity which intermediates between two definitions, acylindricity, due to Sela in the case of trees and Bowditch [Bo], and the weak proper discontinuity property, WWPD due to Bestvina Bromberg and Fujiwara [Be-Br-Fu]. We will need to use the basic properties of isometries of Gromov-hyperbolic spaces ; see for instance [Co-De$\mathrm{Pa}$ ], Chapter 9 for a detailed discussion of the classification and properties of isometries in such a space.

Let us first recall the following definition.

Definition 5 The action of $G$ on a $\delta$-hyperbolic space $H$ is acylindrical, if there exists an integer $N$ and a real $K$ such that for every pair of points $a, b$ with $d(a, b) \geqslant K$ the set $\{g \in G / d(g . a, a)+d(g . b, b) \leqslant 1000 \delta\}$ is finite with cardinality $\leqslant N$.

Let $G$ be a group acting on a $\delta$-hyperbolic space $H$. The translation length $[g]$ of an element $g$ is $[g]=\operatorname{Min}_{x \in H} d(x, g . x)$. Let $g$ be some element with translation length $\geqslant 100 \delta$. Then $g$ is an hyperbolic isometry (see [Co-De-Pa], Lemma 3.1, p. 301) .

More precisely, if $x_{0}$ realizes the minimum $\operatorname{Min}_{x \in H} d(x, g \cdot x)$, the union of geodesic segments $L_{g}=\cup_{n \in \mathrm{Z}} g^{n}\left[x_{0}, g \cdot x_{0}\right]$ is a $[g]$-local geodesic (this fact is true for every geodesic space). In a $\delta$-hyperbolic space such a local geodesic respectively converges as $n$ tends to $+\infty$ and $-\infty$, to two points in the Gromov boundary of $H$, say $g^{+}, g^{-}$, and these two points are fixed by $g$. (This is the definition of a hyperbolic isometry, see [Co-De-Pa], Chap.9).

We consider $L_{g}$ as a $[g]$-local geodesic oriented by the action of $g$. The set $L_{g}$ is called a quasi-line of $g$. Note that a different choice of minimizing point or a different choice of geodesic segment $\left[x_{1}, g . x_{1}\right]$ will give another quasiline $L_{g}^{\prime}$. Using basic properties of Gromov hyperbolic spaces, in particular the fundamental Theorem of "stability of quasi-geodesics" one sees that there exists a universal constant $C$ such that the Hausdorff distance between $L_{g}$ and $L_{g}^{\prime}$ is bounded by $C \delta$.

If $h$ is an hyperbolic element, for instance an element with translation length $[h] \geq 100 \delta$, we denote by $E(h)$ the elementary group defined by $h$, i.e. the subgroup $E(h)=\left\{g \in G / g \cdot\left\{h^{-}, h^{+}\right\}=\left\{h^{-}, h^{+}\right\}\right\}$, where $\left\{h^{-}, h^{+}\right\}$are the two fixed points of $h$ in the boundary of $H$.

Definition 6 The action of $G$ on a $\delta$-hyperbolic space is weakly acylindrical if there exists a real $D>0$ such that for every hyperbolic element $g$, and every $h \notin E(g)$, the diameter of the projection of $h L_{g}$ to $L_{g}$ is bounded by $D(1+[g])$. 
If the constant $D$ is specified, the action is called $D$ - weakly acylindrical

This definition of weak acylindricity is a uniform version of the WWPD property introduced by $[\mathrm{Be}-\mathrm{Br}-\mathrm{Fu}]$ : the WWPD property requires that, there exists a constant $\kappa(g)$ such that for $h \notin E(g)$, the diameter of the same set $\{x \in$ $\left.L_{g} / d(x, h x) \leqslant 100 \delta\right\}$ is bounded by $\kappa(g)$. We require that $\kappa(g) \leq D$. $([g]+1)$.

The following Proposition (which is not obvious despite its formulation) explain the relationship between the two concepts of acylindricity. Its proof is very similar to the usual proof of the Margulis-Zassenhaus Lemma.

Proposition 5 An acylindrical action on a hyperbolic space is weakly acylindrical.

Proof Let $(K, N)$ be the two constants involved in the definition of acylindricity. Let us fix some hyperbolic element $g$, and let $E(g)$ be the elementary subgroup defined by $g$. Let $\Delta$ be the maximum of the diameter of the sets $P_{h}=\left\{a \in L_{g} / \exists a^{\prime} \in h L_{g} / d\left(a, a^{\prime}\right) \leqslant 10 \delta\right\}$ for $h \notin E(g)$. We will prove that $\Delta \leq(N+1)([g]+1000 \delta)+K$, hence the $D$-weak acylindricity for $D=$ $\max (N+1, K+(N+1) 1000 \delta)$.

Let $h \in G$, and let us assume that the curve $L_{g}$ contains a segment $I=[a, b]$ of length $(N+1)([g]+1000 \delta)+K$ such that $\exists a^{\prime}, b^{\prime} \subset h L_{g}$ with $d\left(a, a^{\prime}\right) \leqslant$ $10 \delta, d\left(b, b^{\prime}\right) \leqslant 10 \delta$. We will prove that $h \in E(g)$.

Note that for every $c \in[a, b]$ there exists $c^{\prime} \in\left[a^{\prime}, b^{\prime}\right]$ with $d\left(c, c^{\prime}\right) \leqslant 12 \delta$. Let $a_{1}=g . a$ and $I_{1}=\left[a_{1}, b\right] \subset[a, b]$. As $d\left(a, a_{1}\right)=[g]$, the length of $I_{1}$ is the length of $I$ minus the translation length $[g]$. As $g$ acts as a translation of length $[g]$ on $L_{g}$, its conjugate $h . g \cdot h^{-1}$ acts as a translation of the same length on $h L_{g}$. Therefore, if $h_{1}=h^{-1} g . h \cdot g^{-1}$, for every $x \in I_{1}, d\left(h_{1} x, x\right) \leqslant 20 \delta$. Replacing $g$ by $g^{k}, k \leqslant N+1$, and defining $a_{k}=g^{k} a$ as the point of $[a, b] \subset L_{g}$ with $d\left(a_{k}, a\right)=k[g]$, and $h_{k}=h^{-1} g^{k} h g^{k}$, we see that if $\Delta-(N+1)[g]-100 \delta>0$, then $d\left(a_{N+1}, b\right)>K$, and for every $k$, we have $d\left(h_{k} a_{N+1}, a_{N+1}\right) \leqslant 20 \delta$ and $d\left(h_{k} b, b\right) \leqslant 20 \delta$. Therefore, by acylindricity, the family $\left(h_{k}\right)_{1 \leq k \leq N+1}$ take at most $N$ values, and for two different indexes, $k, l, h^{-1} g^{k} h g^{-k}=h^{-1} g^{l} h g^{-l}$, or $\left[h, g^{k-l}\right]=1$, hence $h \in E(g)$.

Example 1 Let $G$ be a group acting discretely on some simply connected manifold with pinched negative curvature $H$. Let $\delta$ be the hyperbolicity constant, and $\mu$ the Margulis constant of $H$. Let $g \in G$ be an hyperbolic isometry, $A=\{h /$ the diameter of the projection of $h . L_{g}$ to $L_{g}$ is greater than $\left.2[g]+100 \delta+\mu\right\}$. Arguing as in the proof of Proposition 4, and using the Margulis Lemma, one proves that the set $B=\left\{g h g^{-1} h^{-1}, h \in A\right\} \cup\{g\}$ generates a subgroup $K$ such that $[K, K)]$ is nilpotent. The group $K$ is therefore solvable, hence an elementary subgroup of $G$, containing $g$. Therefore if $h \in A$, the commutator $g \cdot h \cdot g^{-1} h^{-1}$ is in $E(g)$, as well as $h g^{-1} h^{-1}$ and $h$ fixes the two fixes point of $g$ at infinity. Note that if there is some parabolic element, the action is not acylindrical, and weak acylindricity is better adapted to this situation. 
In his survey on R-trees [Be2], M. Bestvina explain why Margulis Lemma implies that a limit of discrete faithfull actions on a classical hyperbolic space has virtually nilpotent edge stabilizers. In our case, we have no Margulis Lemma, and the actions are not faithful. However, we will prove that, under some weak acylindricity assumption, the kernel of the action of the limit group on the limit tree is finite; of course our proof is very similar to that of [Be2].

Proposition 6 Let $(H, d)$ be a hyperbolic space, $G$ a group of isometries of $H, \Gamma$ a finitely generated group and $\varphi_{n}: \Gamma \rightarrow G$ a sequence of actions, $x_{n}$ a sequence of base points with $\lim _{\omega} e_{n}\left(x_{n}\right)=\infty$. Let $H_{\omega}=\lim _{\omega}\left(H, d_{n}, x_{n}\right)$ for $d_{n}=\frac{d}{e\left(x_{n}\right)}$ be the limit tree and $T_{\omega} \subset H_{\omega}$ the minimal invariant subtree. Let $\Gamma_{\omega}$ the limit group of this sequence. Assume that the action of $G$ on $H$ is weakly acylindrical, and that $\Gamma_{\omega}$ is not elliptic (i.e. $T_{\omega}$ is not a point).

1. Let $N \subset \operatorname{ker}\left(\varphi_{\omega}\right)$ be a finitely generated subgroup contained in the kernel of the limit action. Let $g$ be an element whose image in $\Gamma_{\omega}$ is hyperbolic. Then for $\omega$-almost every $n, \varphi_{n}(N)$ is contained in $E\left(\varphi_{n}(g)\right)$, the maximal elementary subgroup containing $\varphi_{n}(g)$ of $G$.

2. If $T_{\omega}$ is not line, for $\omega$-almost every $n$, the group $\varphi_{n}(N)$ is elliptic.

3. If $T_{\omega}$ is a line, for infinitely many $n, \varphi_{n}(\Gamma)$ is elementary.

4. Assume that the action of $G$ on $H$ is acylindrical. Then the kernel of the action of $\Gamma_{\omega}$ on $T_{\omega}$ is finite if $T_{\omega}$ is not a point or line, or virtually abelian if $T_{\omega}$ is a line

Proof As $\Gamma$ is finitely generated, and as its action on $T_{\omega}$ is not elliptic, it contains an element $g$ whose image in $\Gamma_{\omega}$ is an hyperbolic isometry of $T_{\omega}$. Let $\Lambda_{g} \subset T_{\omega}$ be the $g$-invariant line, and $[g]_{\omega}$ its translation length. Let us first note that $[g]_{\omega}=\lim _{\omega}\left[\varphi_{n}(g)\right]_{n}$. Indeed, if $m_{n} \in H_{n}$ is a midpoint of a segment $\left[x_{n}, \varphi_{n}(g) x_{n}\right]$, one has that $\left[\varphi_{n}(g)\right] \leqslant d_{n}\left(m_{n}, \varphi_{n}(g) m_{n}\right) \leqslant\left[\varphi_{n}(g)\right]+10 \delta_{n}$. Note that $d_{n}\left(x_{n}, \varphi_{n}(g) x_{n}\right)$ is bounded, therefore $m_{n}$ converges to a point which is a midpoint of $\left[x_{\omega}, g \cdot x_{\omega}\right]$, hence on $\Lambda_{g}$. If $y_{n} \in L_{\varphi_{n}(g)}$ is such that $d_{n}\left(x_{n}, y_{n}\right)$ is bounded, the same argument proves that $\lim _{\omega} y_{n} \in \Lambda_{g}$. As $\lim _{\omega}\left[\varphi_{n}(g)\right]_{n}=[g]$, for $\omega$-almost every $n,\left[\varphi_{n}(g)\right]_{n} \gg \frac{\delta}{e_{n}}=\delta_{n}$, the hyperbolicity constant of $H_{n}$. Note that $g$ acts on its axis $L_{\varphi_{n}(g)}$ as a translation of length $\left[\varphi_{n}(g)\right]_{n}$. Let $h \in \Gamma$, and assume that $\varphi_{\omega}(h)=\operatorname{Id}_{T_{\omega}}$. Let us prove that for $\omega$-almost $n$, $\varphi_{n}(h) \in E\left(\varphi_{n}(g)\right)$. Let $\alpha, \beta$ be two points on $L$ at a distance $>D\left([g]_{\omega}+1\right)+1$. These points are $\omega$-limits of points $a_{n}, b_{n}$ or of $\varphi_{n}(g) a_{n}, \varphi_{n}(g) b_{n}$. In particular, $d\left(a_{n}, \varphi_{n}(g) a_{n}\right)=o\left(e_{n}\right), d\left(b_{n}, \varphi_{n}(g) b_{n}\right)=o\left(e_{n}\right)$, but $d\left(a_{n}, b_{n}\right) \geqslant D\left(\left[\varphi_{n}(g)\right]+\right.$ $1)+e_{n}$. Let $a_{n}^{\prime}$ and $b_{n}^{\prime} \in\left[a_{n}, b_{n}\right]$ be the points at distance $e_{n}$ from $a_{n}$ and $b_{n}$ respectively. Then for $\omega$-almost every $n$, the distance between the point $a_{n}^{\prime}$ (respectively $b_{n}^{\prime}$ ) and $\varphi_{n}(g)\left[a_{n}, b_{n}\right]$ is bounded above by $10 \delta$. By (weak) acylindricity $\varphi_{n}(h) \in E\left(\varphi_{n}(g)\right)$. This proves the first point of the proposition.

If $T_{\omega}$ is not a line, for some $u$ the line $\Lambda_{g}$ is not stable by $u: \Lambda_{u . g . u^{-1}}=$ $h_{\omega}(u) \Lambda_{g} \neq \Lambda_{g}$. Furthermore, if $N$ is a f.g. subgroup of the kernel of the action, for $\omega$-almost all $n, \varphi_{n}(N) \subset E\left(\varphi_{n}\left(u g u^{-1}\right)\right)$ (by applying the argument 
of 1 to $\left.u . g . u^{-1}\right)$. Then, the group $\varphi_{n}(N)$ is contained in the two elementary subgroups generated by $\varphi_{n}(g)$ and $\varphi_{n}\left(u \cdot g \cdot u^{-1}\right)$. It is thus elliptic, and contained in $E\left(\varphi_{n}(g)\right)$. This proves (2). In particular it is finite in the acylindrical case (4).

If the limit space is a line, $h$ acts with non zero translation length on this line, and $\sigma \in \Sigma$, we can apply (1) to the commutator $\sigma h \sigma^{-1} h^{-1}$ : this element is in $E\left(\left(\varphi_{n}(h)\right)\right.$ for $\omega$-almost all $n$, and as $\Sigma$ is finite, this prove 3 .

\subsection{Kähler groups.}

Let us keep the notations of Section 3.3 unchanged, but assume now further that $\Gamma$ is a Kähler group ; applying Theorem 3 and Proposition 6 we get :

Theorem 6 Let $\Gamma$ be a Kähler group; let $G$ be a group of isometries of a hyperbolic space $(H, d), \varphi_{n}: \Gamma \rightarrow G$ a sequence of actions, $x_{n} \in H$ a sequence of base points with energy $\lim _{\omega} e_{n}\left(x_{n}\right)=\infty$. Let $H_{\omega}=\lim _{\omega}\left(H, \frac{1}{e_{n}} d_{n}, x_{n}\right)$, and $T_{\omega} \subset H_{\omega}$ be the minimal invariant subtree, and assume that the limit action is not elliptic.

1. If the limit action is not elementary, then $\Gamma$ fibers. There exists a 2orbifold $\Sigma$ and a surjective homomorphism $\pi: \Gamma \rightarrow \pi_{1}^{\text {orb }}(\Sigma)$ with finitely generated kernel $N$. The same is true if $T_{\omega}$ is a line, but the kernel of the action of $\Gamma$ on this line is not finitely generated.

2. If the action of $G$ on $H$ is weakly acylindrical, and $T_{\omega}$ is not a line, then for $\omega$-almost every $n$, the group $\varphi_{n}(N)$ is a finitely generated normal subgroup of $\varphi_{n}(G)$ contained in an elliptic subgroup of $G$.

3. If the action of $G$ on $H$ is weakly acylindrical, and $T_{\omega}$ is a line, for $\omega$-almost all $n, \varphi_{n}(\Gamma)$ is contained in an elementary subgroup of $G$.

4. If the action of $G$ on $H$ is acylindrical, then for $\omega$-almost every n, $\varphi_{n}(N)$ is a finite group. Therefore $\varphi_{n}$ factors, via $\pi$, through a finite extension of $\pi_{1}^{\text {orb }}(\Sigma)$.

Proof 1. If the action of $\Gamma$ on the limit tree is not elementary, Theorem 3 (or Theorem 4) applies. Then the second point follows from Proposition 6 (2), the third point from Proposition 6 (3), and the last one from Proposition 6 (4).

Example 2 This Theorem implies a compactness result on non elementary discrete actions on a simply connected manifold with pinched non positive curvature, for instance the complex or quaternionic hyperbolic space : such an action is weakly acylindrical (Example 1). Let $G=\operatorname{Isom}(H)$ where $H$ is a complete simply connected manifold with pinched strictly negative curvature. As $\Gamma$ is finitely generated the set $\operatorname{Hom}_{\mathrm{disc}}(\Gamma, G) /$ conj of conjugacy classes of discrete non elementary representations of $\Gamma$ to $G$ is endowed with a natural topology, such that the set of representations of bounded energy is compact. Our result 
(2) applies. Furtheremore, in a Kleinian group, more generally if $H$ is a CAT(-1) space, a f.g. elliptic group induces the identity on a totally geodesic subspace, reducing the dimension. Thus, the set of non elementary representations which do not factor through the fundamental group of a 2-orbifold and do not preserve a strict totally geodesic subspace (Zariski dense representations in the case of rank 1 symmetric spaces) is a compact subset of $\operatorname{Hom}_{\text {disc }}(\Gamma, G)$. Note that, in the case of $\mathrm{SO}(n, 1)$ this set is even known to be empty (see [Ca-To]) if $n \geq 3$. It would be interesting to have an arithmetic interpretation of the finite set of connected components of this representations spaces, in the spirit of [Co-Si].

If $G=G=\mathrm{SU}(n, 1)$ or $\mathrm{Sp}(n, 1)$, and $\Gamma=\pi_{1}(X), X$ Kähler, the results of Siu [Si] (improved by Carlson and Toledo [Ca-To]) implies that a representation of $\Gamma$ in $G$ which do not factors through a Riemann surface can be realized as an equivariant holomorphic (or anti-holomorphic) map from the universal cover of $X$ to the symmetric space $G / K$. Such a map is 1-Lipshitz for the Kobayashi pseudo distance on $X$, giving an explicit bound on the energy : if $L$ is the greatest Kobayashi length of the generators of $\Gamma$, the energy of the representation that can be realized by an holomorphic map is $\leq L$; a stronger result than our (non effective) compacity result.

\section{Projection complexes and weak acylindricity.}

Let us review the construction of M. Bestvina, K. Bromberg and K. Fujiwara of a projection complex of $\delta$-hyperbolic metric spaces ; we follow the notations of $[\mathrm{Be}-\mathrm{Br}-\mathrm{Fu}]$.

\subsection{Introduction.}

Let $\boldsymbol{Y}$ be a set of $\delta$-hyperbolic geodesic metric spaces. If $Y \in \boldsymbol{Y}$ it is convenient to make a difference between $Y$ as an element of $\boldsymbol{Y}$ and $C(Y)$ the metric space it represents (in our case this will be a graph, in fact a curve complex). We emphasize that the hyperbolicity constant $\delta$ is uniform for $C(Y), Y \in \boldsymbol{Y}$. A function $\pi_{Y}$ is given, called the projection, from $\boldsymbol{Y}-\{Y\}$ to the set of subsets of $Y$ of diameter $\leqslant \theta$. Here $\theta$ is a fixed constant. One extends this function $\pi_{Y}$ on $Y$ by setting $\pi_{Y}(x)=x$ and on the union of $X \in \boldsymbol{Y}-\{Y\}$, by setting $\pi_{Y}(x)=$ $\pi_{Y}(X)$. We assume that the function $d_{Y}^{\pi}(X, Z)=\operatorname{diam}\left\{\pi_{Y}(X) \cup \pi_{Y}(Z)\right\}$ endows $\boldsymbol{Y}$ with the structure of a projection complex in the sense of [Be-Br-Fu], i.e. satisfies two further axioms : for every $A, B, C$, at most one of the three numbers $d_{A}^{\pi}(B, C), d_{B}^{\pi}(C, A)$ and $d_{C}^{\pi}(A, B)$ is greater than $\theta$, and for every $A, B$, the set $\left\{C / d_{C}^{\pi}(A, B)>\theta\right\}$ is finite.

Unfortunately this number $d_{Y}^{\pi}(X, Z)$ does not behave like a hyperbolic distance, and this forces [Be-Br-Fu], Definition 3.1. to introduce a modified distance with better properties.

This new distance is defined in two steps. 
First one define $\mathcal{H}(X, Z)$ as the set of pairs $\left(X^{\prime}, Z^{\prime}\right)$ with $X^{\prime} \neq Z^{\prime}$ and one of the following properties holds.

$$
\begin{aligned}
& \text {-both } d_{X}^{\pi}\left(X^{\prime}, Z^{\prime}\right)>2 \theta, d_{Z}^{\pi}\left(X^{\prime}, Z^{\prime}\right)>2 \theta \\
& -X=X^{\prime} \text { and } d_{Z}^{\pi}\left(X, Z^{\prime}\right)>2 \theta \\
& -Z=Z^{\prime} \text { and } d_{X}^{\pi}\left(X^{\prime}, Z\right)>2 \theta \\
& -X=X^{\prime}, Z=Z^{\prime}
\end{aligned}
$$

Then on defines $d_{Y}(X, Z)=\operatorname{Inf} f_{\left(X^{\prime}, Z^{\prime}\right) \in \mathcal{H}(X, Z)} d_{Y}^{\pi}(X, Z)$.

If $K$ is a given positive constant, let $Y_{K}(X, Z)=\left\{Y / d_{Y}(X, Z)>K\right\}$, and let $P_{K}(\boldsymbol{Y})$ be the graph whose vertex set is $\boldsymbol{Y}$ and where two vertices are joined by an edge if $Y_{K}(X, Z)=\varnothing$.

The first important result of [Be-Br-Fu] (Theorem 3.16) is that for sufficiently large $K$, this metric space is a quasi-tree, i.e. a metric space that is quasiisometric to a tree. In particular, it is a Gromov-hyperbolic space. It appears to be $\beta$-hyperbolic for a universal constant $\beta$. In fact this space satisfies the so-called "bottleneck" property: for every pair of points $a, b$ and $c \in[a, b]$, every continuous path between $a$ and $b$ contains a point $c^{\prime}$ with $d\left(c, c^{\prime}\right) \leqslant 2$. The exact value of the hyperbolicity constant $\beta$ is not meaningful, but it is important that it remains constant as $K$ increases. As the bottleneck constant of $P_{K}(\boldsymbol{Y})$ is 2 , we can choose $\beta=10$.

In a second step, [Be-Br-Fu] construct a "quasi-tree of metric spaces" $C(\boldsymbol{Y})$. This metric space is obtained by taking the disjoint union of the metric spaces $C(Y)$, for $Y \in \boldsymbol{Y}$, and adding an edge of length $L$ from every point of $\pi_{X}(Z)$ to every point in $\pi_{Z}(X)$, if $[X, Z]$ is an edge of $P_{K}(\boldsymbol{Y})$. In this new metric space $C(\boldsymbol{Y})$, the subsets $C(Y)$ are totally geodesically embedded subspaces. We see now that is was important, in the notations, to distinguish between $Y$ as a point in $\boldsymbol{Y}$, and $C(Y)$ a metric space totally geodesically embedded in $C(\boldsymbol{Y})$.

As all metric spaces $Y \in \boldsymbol{Y}$ are uniformly $\delta$-hyperbolic, if $K$ is large enough and $L$ is correctly chosen between $K$ and $2 K$, there exists a constant $\Delta$ such that the metric space $C(\boldsymbol{Y})$ is $\Delta$-hyperbolic [Be-Br-Fu] (Theorem 4.17). Moreover this number $\Delta$ is independent of the choice of $K$. If necessary, we will write $C_{K}(\boldsymbol{Y})$ to remind the dependence on the parameter $K$.

Assume that $G$ is a group acting on $\boldsymbol{Y}$, preserving the maps $\pi$, and such that for every $Y \in \boldsymbol{Y}$ and $g \in G$, there is map $F_{g}: \cup C(Y) \rightarrow \cup C(Y)$, which for each $Y$ restricts to an isometry $F_{g}: C(Y) \rightarrow C(g . Y)$, and such that for all $g, h$ $F_{g . h}=F_{g} . F_{h}$. Then, the group $G$ acts isometrically on $C(\boldsymbol{Y})$.

The following definition, the $\nu$-invariant, is useful to describe algebraic properties of elementary subgroups of certain hyperbolic groups (compare [Co]). The finiteness of $\nu$ can substitute the existence of the Margulis constant when there is no lower bound on the curvature.

Definition 7 Let $G$ act on a set $\boldsymbol{Y}$. One says that $\nu(G, \boldsymbol{Y}) \leqslant \nu$ if the following property holds. Let $h, g \in G$. Suppose that $<h, g \cdot h \cdot g^{-1}, g^{2} . h . g^{-2}, \ldots, g^{\nu} . h . g^{-\nu}>$ fixes a point $Y_{0} \in \boldsymbol{Y}$. Then for every $n \in \mathrm{N}, g^{n} \cdot h \cdot g^{-n}$ fixes this point. 
Proposition 7 Assume that there exists a constant $D$ such that for every $Y \in$ $\boldsymbol{Y}$, the action of the stabilizer $G_{Y}$ on $C(Y)$ is D-weakly acylindrical and assume that $\nu(G, \boldsymbol{Y})=\nu$ is finite. Then, for $K$ large enough, the action of $G$ on $C_{K}(\boldsymbol{Y})$ is weakly acylindrical.

In order to prove this Proposition, we will study the action of $G$ on the metric space $C_{K^{*}}(\boldsymbol{Y})$, where $K^{*}$ will be defined later, in the proof of Lemma 3 in 4.3. Recall that $\Delta$ is a fixed common hyperbolicity constant for the metric spaces $C_{K^{\times}}(\boldsymbol{Y})$ provided $K^{\times} \geqslant K$. Let $g \in G$ be an element acting on $C_{K^{*}}(\boldsymbol{Y})$ and assume that $g$ is a hyperbolic isometry. Up to replacing $g$ by some power, we may assume that the translation length $[g]$ of $g$ is large compared to the hyperbolicity constant: $\operatorname{Min}_{x \in C_{K^{*}(y)}} d(x, g \cdot x)=[g]_{C_{K^{*}}(\boldsymbol{Y})} \geqslant 10^{4} \Delta$. If $L_{g}$ is a quasi-line of $g$ and $h \notin E(g)$, we have to estimate the diameter of the projection of $h . L_{g}$ to $L_{g}$. The meaning of Proposition 7 is that this diameter is bounded by a constant time the translation length of $g$.

In order to estimate this diameter, we will distinguish two very different cases in the next two sections (4.2 and 4.3 respectively). If the isometry $g$ is hyperbolic for its action on $P_{K^{*}}(\boldsymbol{Y})$, we will see that it has an axis when acting on $P_{K}$. If it is elliptic or parabolic, then we will see that it fixes a point in $\boldsymbol{Y}$. We remark that both cases have been already discussed in [Be-Br-Fu], and their estimates will imply the result.

\subsection{Elliptic or parabolic case.}

Recall that for all $K$ large enough, the graph $P_{K}(\boldsymbol{Y})$ is 10-hyperbolic. Before stating the main result of this subsection let us state and prove a Lemma (well known but hard to find in the literature) about non-hyperbolic isometries of hyperbolic spaces.

Lemma 1 If $H$ be a hyperbolic space, $g$ an isometry of $H$. If $g$ is elliptic, there exists a point in $H$ such that $\forall n \in \mathrm{Z}, d\left(g^{n} x_{0}, x_{0}\right) \leq 5 \delta$. If $g$ is parabolic, for every integer $N_{0}$, there exists a point $x_{0}$ such that $\forall n \in\left\{1,2, . ., N_{0}\right\}, d\left(g^{n} x_{0}, x_{0}\right) \leq$ $10 \delta$

Proof Assume firstly that $g$ is elliptic, let $x_{1} \in H$ its $g$-orbit $B$ is bounded, and let $\rho=\in\{r \in \mathrm{R} / \exists x / B \subset B(x, r)\}$ its radius. We know ([Br-Ha], Lemma 3.3 page 460), that the $g$ invariant set $\{c B \subset B(c, \rho+\delta / 2)\}$ is of diameter less than $5 \delta$. The orbit of any point $x_{0}$ in this set satisfy our property.

Assume now that $g$ is parabolic and let $p$ be a fixed point at infinity. Recall that a ray is a geodesic $r:[0, \infty[\rightarrow X$ parametrized by arc length, and that two rays ending at the same point in $\partial X$ must be $2 \delta$-close outside of a bounded set (thinness of triangles). We will prove that if $t$ is large enough, if $x_{0}=r(t)$ $\forall n \in\left\{1,2, . ., N_{0}\right\}, d\left(g^{n} x_{0}, x_{0}\right) \leq 10 \delta$; of course $t$ depends on $N_{0}$. The example of the translation of the hyperbolic plane $g(z)=z+1$ is a good illustration: every point in the ray $\operatorname{Re}(z)=0, \operatorname{Im}(z)>\frac{1}{N_{0}}$ satisfies $\forall n \in\left\{1, . ., N_{0}\right\}, d\left(g^{n} \cdot x, x\right) \leq 1$. 
As $g$ is not hyperbolic, there exists a point $x_{1}$ with $d\left(x_{1}, g x_{1}\right) \leq 6 \delta$ ([CoDe-Pa], Lemma 3.1, page 101). Let us choose a ray $r_{1}$ starting at $x_{1}$ and ending at $p$. As $p$ is fixed by $g$, there exists at $t_{1}$ such that for every $t>t_{1}$, $g . r_{1}(t)$ is $2 \delta$-close to the ray $r_{1}$. By the triangular inequality, if $t>t_{1}$ we have $d\left(r_{1}(t), g \cdot r_{1}(t)\right) \leq 6 \delta+2 \delta=8 \delta$.

As two rays $r, r^{\prime}$ ending at $p$ are $2 \delta$-close outside a compact set, we see that for every ray $r:\left[0, \infty\left[\rightarrow X\right.\right.$ ending at $p$ there exists a $t_{c}$ such that if $t>t_{c}, d(g \cdot r(t), r(t)) \leq 10 \delta$. One fixes a ray $r$ ending at $p$. As $g^{n}$ fixes $p$, for every $n \leq N_{0}$ we can find $t_{n}$ such that if $t>t_{n}, d(g . r(t), r(t)) \leq 10 \delta$. In particular any point $x_{0}$ in the geodesic ray $r\left(\left[\sup t_{n},+\infty[\right.\right.$ satisfies our property.

Lemma 2 If $g$ on $P_{K^{*}}(\boldsymbol{Y})$ the element $g$ is elliptic or parabolic, then $g$ fixes a point in $\boldsymbol{Y}$.

Proof As $P_{K^{*}}(\boldsymbol{Y})$ is 10-hyperbolic, for every $N_{0}$, one can find a point in this graph (i.e an element $Y_{0} \in \boldsymbol{Y}$ ), such that $d\left(Y_{0}, g^{n} Y_{0}\right) \leqslant 100$ for all $1 \leqslant n \leqslant N_{0}$.

More precisely, Let $n$ be a fixed integer ( $n$ will be chosen later) and consider a geodesic path of length $\leqslant 100$ between $Y_{0}$ and $g^{n} Y_{0}$. This path can be written as a sequence of adjacent vertices $Y_{0}=X_{0}, X_{1}, \ldots X_{k}=g^{n} X_{0}$ with $k \leqslant 100$. For every $i \in\{0,1, . ., k-1\}$, one chooses a point $x_{i}^{+}$in $\pi_{X_{i}}\left(X_{i+1}\right)$ (the diameter of this set is $\leqslant \theta$ ) and for $i \in\{1, . ., k\}$, a point $x_{i}^{-}$in $\pi_{X_{i}}\left(X_{i-1}\right)$. One sets $x_{n}^{+}=g^{n} x_{0}$, and for every $i \in\{1, \ldots, k\}$, one considers a geodesic segment $\left[x_{i-}, x_{i^{+}}\right]$in $X_{i}$. Note that, for every $i, d\left(x_{i}^{+}, x_{i+1}^{-}\right) \leqslant L^{*}$, and that $\left[x_{i}^{+}, x_{i+1}^{-}\right]$is an edge of $P_{K^{*}}(\boldsymbol{Y})$. We consider the path $\gamma=\left[x_{0}^{+}, x_{1}^{-}\right] \cup\left[x_{1}^{-}, x_{1}^{+}\right] \cup \ldots\left[x_{k}^{-}, x_{k}^{+}\right]$. We remark that if $g^{n} Y_{0}=Y_{0}$, this path is entirely contained in $Y_{0}$.

Applying [Co-De-Pa] Lemma 1.5 page 25 , one gets that in $C_{K^{*}}(\boldsymbol{Y})$ any geodesic $\left[x_{0}^{+}, g^{n} \cdot x_{0}\right]$ remains in the $\left(\ln _{2} k\right) \Delta$-neighborhood (or $10 . \Delta$ as $k \leqslant 100$ ) of this path $\gamma$.

Let $y_{0}$ be a point where the function $d(y, g y)$ is minimal, equivalently $[g]=$ $d\left(y_{0}, g . y_{0}\right)$. By minimality, the $g$-invariant set $L_{g}=\cup_{k \in \mathrm{Z}} g^{k}\left[y_{0}, g . y_{0}\right]$ is a $[g]$ local geodesic (a quasi-line) and is therefore $100 \Delta$-quasi-convex ([Co-De-Pa] Proposition 10.3.1). Let $p$ be a projection of $x_{0}$ on this set ( $p$ realizes the minimal distance of $x$ to $\left.L_{g}\right)$. Then $q=g^{n} p$ is at the distance $\geqslant n([g]-100 \Delta)$ of $p$. Therefore the segment $\left[q, g^{n} q\right]$ of $L_{g}$ contains a subsegment $[a, b]$ of length $\geqslant$ $\frac{n([g]-100 \Delta)-L^{*}}{K}$ which is $10 \Delta$-close to a segment $\left[x_{i}^{-}, x_{i}^{+}\right]$. Let $A$ be some positive number. Choosing $n$ large enough, one may assume that $d(a, b) \geqslant[g]+A$ and find an arbitrary long segment (of size $>A$ ) in some metric space $X_{i}$ whose image by $g$ is $10 \Delta$-close to $X_{i}$. Choosing $A>\theta+1000 \Delta$ (and $n$ consequently), we see that the projection of $g X_{i}$ to $X_{i}$ has an arbitrary large diameter : by definition of a projection complex, the point $X_{i}$ is fixed by $g$. This proves Lemma 2. 
Now, in order to study the action of $g$ on $C_{K}(\boldsymbol{Y})$ we can use the argument of [Be-Br-Fu], Proposition 4.20. The metric space $X_{i}$ is a totally geodesic subspace of $C_{K}(\boldsymbol{Y})$ fixed by $g$, and $L_{g} \subset X_{i}$ is a $g$-invariant $[g]$-local geodesic. If the diameter of the projection of $h . L_{g}$ on $L_{g}$ is greater than $\theta$, then $h$ must fix $X_{i}$. This implies that $E(g) \subset G_{X_{i}}$. Then, the $D$-acylindricity of the action of $G_{X_{i}}$ on $X_{i}$ implies that if $h \notin E(g)$ but $h \in G_{X_{i}}$, the diameter of the projection of $h . L_{g}$ to $L_{g}$ is bounded by $D(1+[g])$. Summing up:

(1) If, acting on $P_{K^{*}}(\boldsymbol{Y}), g$ is elliptic or parabolic and if the diameter of the projection of $h . L_{g}$ on $L_{g}$ is greater than $\theta+D(1+[g])$, then $h \in E(g)$.

\subsection{Hyperbolic case.}

Let us now assume that $g$ is a hyperbolic isometry for its action on $P_{K^{*}}$. Recall that an axis of $g$ is a $g$-invariant subset of a bi-infinite geodesic (joining the two fixed points of $g$ at infinity). It is contained in a bi-infinite geodesic, but might be a proper subset.

If a point $X$ belongs to every bi-infinite geodesic between the two fixed points at infinity, we call it stable $([\mathrm{Be}-\mathrm{Br}-\mathrm{Fu}])$. The set of stable points (if not empty) is an axis, invariant by $E(g)$. We call it the stable axis of $g$ (if it is not empty). If $X_{0}$ belongs to the stable axis of $g$, if $X_{1}=g X_{0}$ is the $g$ orbit of $X_{0}$ and $\left[X_{0}, X_{1}\right]$ is a fixed geodesic segment, then the union $\cup_{n \in \mathrm{Z}} g^{n}\left[X_{0}, X_{1}\right]$ is a $g$-invariant geodesic. The following Lemma is very similar to Lemma 3.22 from [Be-Br-Fu].

Lemma 3 1. In the metric space $P_{K}$, the isometry $g$ has a stable axis $\alpha$.

2. If furthermore $h \in G$ is such that the diameter (measured in $P_{K}$ ) of the projection of h. $\alpha$ on $\alpha$ is greater than $(k+1)[g]+1000$, then this stable axis contains a point $X_{0}^{\prime}$ such that for every $p \in\{0, \ldots k\}$, the commutator $h^{\prime}=h g^{-1} h^{-1} g$ fixes $g^{p} X_{0}^{\prime} \in \alpha$.

3. If furthermore $k \geqslant \nu(G, \boldsymbol{Y})$, then $h$ belongs to the elementary (for the action on $P_{K}$ ) subgroup of $G$ containing $g$.

Proof As $g$ is hyperbolic for its action on $P_{K^{*}}$, up to replacing $g$ by some power, one can assume that its translation length $d=\operatorname{Min}_{Y} d(Y, g Y)$ is greater than $10^{5}$ ( $10^{4}$ times the hyperbolicity constant which is $\left.\leq 10\right)$. Let $X_{0}$ be chosen so that in the graph $P_{K^{*}}, d\left(X_{0}, g X_{0}\right)=d$ is minimal. Let $X_{1}=g \cdot X_{0}$, and let $\left[X_{0}, X_{1}\right]$ be a geodesic segment in the graph $P_{K^{*}}$ between these points. We consider a geodesic path $\left\{X_{0}=Y_{0}, Y_{1}, \ldots Y_{d}=X_{1}\right\}=\left[X_{0}, X_{1}\right]$ between these two points . By isometry, if $Y_{-i}=g^{-1} Y_{n-i}$, the path $\left\{X_{-1}=Y_{-d}, . Y_{-d+1}, \ldots Y_{-1}, Y_{0}=X_{0}\right\}$ is a geodesic segment between $X_{-1}=g^{-1} X_{0}$ and $X_{0}$. Let $X_{n}=g^{n} X_{0}$, and $Y_{n . d+k}=g^{n} Y_{k}$. Note that by minimality the sequence $\left(Y_{j}\right)_{j \in \mathrm{Z}}$ is a $d$-local geodesic path in $P_{K^{*}}$. Let $k \leqslant \frac{n}{2}$. As $d\left(Y_{-k}, Y_{k}\right)=2 k$, the set $Y_{K^{*}}\left(Y_{-k}, Y_{k}\right)=$ $\left\{Z / d_{Z}\left(Y_{-k}, Y_{k}\right) \geqslant K^{*}\right\} \cup\left\{Y_{-k}, Y_{k}\right\}$ is another (injective) path of length $\geqslant 2 k$ 
([Be-Br-Fu] proof of Proposition 3.7). By the 10-bottelneck property ([Be-Br$\mathrm{Fu}$ ] Theorem 3.16), this path contains a point $X_{0}^{\prime}$ which is 10-close to $X_{0}$. Now $d\left(X_{0}^{\prime}, Y_{l}\right) \geqslant l-10 \geqslant 3$ if $|l| \geqslant 13$. Therefore, applying ([Be-Br-Fu] Proposition 3.14) to the path $\left\{Y_{l}, Y_{l+1}, \ldots Y_{n}\right\}$ and the vertex $X_{0}^{\prime}$, one obtains that if $13 \leqslant l \leqslant n$, then $d_{X_{0}^{\prime}}\left(Y_{l}, Y_{n}\right)$ as well as $d_{X_{0}^{\prime}}\left(Y_{-l}, Y_{-n}\right)$ is bounded by a constant $c_{\theta}$ depending only on $\theta$. By the triangle inequality, if $l, m \geqslant 13$, then $d_{X_{0}^{\prime}}\left(Y_{-l}, Y_{m}\right) \geqslant K^{*}-2 c_{\theta}$. In particular if $K^{*}$ is sufficiently large, then for every $p, q \geqslant 1, d_{X_{0}^{\prime}}\left(g^{-p} X_{0}, g^{q} X_{0}\right)>K^{\prime}$, where $K^{\prime}$ is the constant introduced in Lemma 3.18 of [Be-Br-Fu]. Similarly, let $l, q>200$ and consider a pair of points $\left\{A_{-p}, A_{q}\right\}$ such that $d\left(A_{-p}, Y_{-p}\right) \leqslant 100$ and $d\left(A_{q}, Y_{q}\right) \leqslant 100$. Let $\left[A_{q}, Y_{q}\right]$ and $\left[A_{-p}, A_{q}\right]$ be two geodesic paths. Then the paths $\left\{Y_{-l}, Y_{l+1}, \ldots Y_{-p}\right\} \cup\left[A_{-p}, Y_{-p}\right]$ and $\left\{Y_{l}, Y_{l+1}, \ldots Y_{q}\right\} \cup\left[A_{q}, Y_{q}\right]$ remain at a distance at least 3 from $X_{0}^{\prime}$. Moreover if $K^{*}$ is sufficiently large then $d_{X_{0}^{\prime}}\left(A_{-p}, A_{q}\right)>K^{\prime}$.

The Lemma 3.18 of [Be-Br-Fu] applies : in $P_{K}(\boldsymbol{Y})$ for every $p, q \geqslant 0$, the point $X_{0}^{\prime}$ belongs to every geodesic between $g^{-p} X_{0}$ and $g^{q} X_{0}$, and even between the two points $A_{-p}, A_{q}$.

By equivariance, for every integer $n$, the point $g^{n} X_{0}^{\prime}$ belongs to every geodesic between $g^{-l} X_{0}$ and $g^{m} X_{0}$ if $l, m>2 n$, and every geodesic segment $\left[A_{-l}, A_{m}\right]$ between two points $A_{p}, A_{q}$ such that $d\left(A_{p}, g^{p} X_{0}^{\prime}\right) \leqslant 100$. All the points $g^{n} X_{0}^{\prime}$ are therefore on a $g$-invariant geodesic $\alpha$. In fact, the same argument shows that for every geodesic $\beta$ parallel to $\alpha$, the point $X_{0}^{\prime}$ belongs to $Y_{K^{\prime \prime}}(\beta)$. Thus Corollary 3.19 from [Be-Br-Fu] proves that $X_{0}^{\prime}$ belongs to every geodesic $\beta$ which is parallel to $\alpha$ : the isometry $g$ has a stable axis, and the first point of Lemma 2 is established.

For the second assertion, note that the hypothesis proves that one can find two points $P, Q$ on $\alpha$ such that the distance between $P$ or $Q$ and the geodesic $h \alpha$ is bounded by 100 , but $d(P, Q)>(\nu+1)[g]+200$. Let $k \leqslant \nu$. The length of the segment $[P, Q]$ being greater than $(k+1)[g]+200,[P, Q]$ contains at least $k+1$ consecutive translates of $X_{0}^{\prime}$. Reordering them, we may assume that these points are $X_{0}^{\prime}, g X_{0}^{\prime}, \ldots, g^{k} X_{0}^{\prime}$. By hyperbolicity, these points are 100-close to some points $Z_{0}, Z_{1}, Z_{k}$ on $h \alpha$, chosen in such a way that $d\left(Z_{i}, Z_{i+1}\right)=[g]$. Thus $h^{-1} Z_{i} \in \alpha$, and $g h^{-1} Z_{i}=h^{-1} Z_{i+1}$, as $d\left(h^{-1} Z_{i}, h^{-1} Z_{i+1}\right)=[g]$. Since $d\left(Z_{0}, X_{0}^{\prime}\right) \leqslant 100$ and $d\left(Z_{2}, g^{2} X_{0}^{\prime}\right) \leqslant 100$, every geodesic between $Z_{0}$ and $Z_{2}$ must go through $g X_{0}^{\prime}$. Replacing $Z_{0}$ by a point $Z_{0}^{\prime} \in h \alpha$ such that $d\left(Z_{0}^{\prime}, g X_{0}^{\prime}\right)=[g]$, and setting $Z_{i}^{\prime}=h g^{i} h^{-1} Z_{0}^{\prime}$ we now have $Z_{i}^{\prime}=g^{i} X_{0}^{\prime}$ for $i \in\{1, \ldots, k\}$. Let $h^{\prime}=h g^{-1} h^{-1} g$. For every $i \in\{0, \ldots k-1\}, h^{\prime} \cdot g^{i} X_{0}^{\prime}=h g^{-1} h^{-1} g^{k+i+1} X_{0}^{\prime}=$ $h g^{-1} h^{-1} Z_{i+1}^{\prime}=Z_{i}^{\prime}=g^{i} X_{0}^{\prime}$. The points $\left(g^{i} X_{0}^{\prime}\right)_{0 \leqslant i \leqslant k-1}$ are fixed by $h^{\prime}$. The second assertion is proved.

By definition of the $\nu$-invariant, if $k \geqslant \nu$, the isometry $h^{\prime}$ fixes $g^{n} X_{0}^{\prime}$ for all $n$. Therefore $h^{\prime}=h \cdot g \cdot h^{-1} g^{-1}$ is in the elementary subgroup of $g$, as well as $h . g . h^{-1}$, and $h$. The third assertion and the Lemma are proved.

In order to complete the proof of Proposition 7, we consider an element $g$ which is a hyperbolic isometry for its action on $P_{K^{*}}$. 
Recall that $\Delta$ denotes a hyperbolicity constant of $C_{K}(\boldsymbol{Y})$. Replacing $g$ by some power, we may assume that $[g]>1000 \Delta$. Let $y_{0} \in C(\boldsymbol{Y})$ be a point such that $d\left(y_{0}, g . y_{0}\right)$ is minimal, and $\left[y_{0}, g . y_{0}\right]$ a geodesic segment. Hence $L_{g}=$ $\cup_{n \in \mathrm{Z}} g^{n}\left[y_{0}, g \cdot y_{0}\right]$ is a quasi-line of $g$, which is a $g$-invariant $[g]$-local geodesic. Let $y_{n}=g^{n} y_{0}$, and $Y_{n}=g^{n} Y_{0} \in \boldsymbol{Y}$ be the unique point such that $y_{n} \in Y_{n}$. Let $\alpha$ be a $g$-invariant axis of $g$ for its action on $P_{K}$ (it exists due to Lemma 6 ), let $X_{0} \in \alpha$ be a projection of $Y_{0}$. By equivariance $X_{n}=g^{n} \cdot X_{0}$ is a projection of $Y_{n}$. Let $h \in E(g)$. Assume that the diameter of the projection of $h . L_{g}$ to $L_{g}$ is at least $(\nu+4)[g]+1000 \Delta$. One can find four points $a, b, c, d$ in $L_{g}$ such that $d(a, h . c) \leqslant 10 \Delta, d(b, h . d) \leqslant 10 \Delta$, and $d(a, b) \geqslant(\nu+4)[g]$. As every point in $L_{g}$ belongs to some segment $\left[y_{i}, y_{i+1}\right]$, we even can find 4 integers $\alpha, \beta, \gamma, \delta$ such that $d\left(y_{\alpha}, h y_{\gamma}\right) \leqslant 10 \Delta, d\left(y_{\beta}, h . y_{\delta}\right) \leqslant 10 \Delta$, and $\beta-\alpha=\delta-\gamma>(\nu+2)$.

Now, if in $C_{K}(\boldsymbol{Y})$ two points $y, z$ are $10 \Delta$-closed, the distance of their images $Y, Z$ in the complex $P_{K}$ is bounded by a uniform constant, $d(Y, Z) \leqslant$ $\frac{10 \Delta}{L}$. Therefore, if $[g] \gg \frac{10 \Delta}{L}$ and if $Y_{i}$ is the space containing $y_{i}$, one has $d\left(Y_{\alpha}, h Y_{\gamma}\right)+d\left(Y_{\beta}, h . Y_{\delta}\right) \leqslant \frac{1}{10}[g]$. A similar inequality occurs for the projections $X_{\alpha}, X_{\beta}, X_{\gamma}, X_{\delta}$ of these points on the stable axis $\alpha$ of $g$ : in a $\Delta$-hyperbolic space projection decreases distances up to an error of $12 \Delta$ (see [Co-De-Pa] Proposition 2.1 p.108). Hence the axis $\alpha$ and its $h$ translate $h \alpha$ are $10 \Delta-$ close along a segment of length $\geqslant(\beta-\alpha-1)[g] \geqslant(\nu+2)[g]$. Applying Lemma 2 , one deduce that the commutator $\left[h, g^{-1}\right]$ fixes the axis of $g$ in $P_{K}$. Therefore in $C(\boldsymbol{Y})$ $\left[h, g^{-1}\right]$ transforms the quasi-line $L_{g}$ in a parallel quasi-line, and $h$ belongs to the elementary subgroup generated by $g$. This proves Lemma 3.

Summing up we proved (2), that if acting on $P_{K^{*}}(\boldsymbol{Y}), g$ is hyperbolic and if the diameter of the projection of $h . L_{g}$ on $L_{g}$ is greater than $\left.(\nu+4)[g]\right)+1000 \Delta$, then $h \in E(g)$.

The combination of (1) and (2) proves the Proposition 7.

\section{Mapping class group, complex of curves and holomorphic families of Riemann surfaces}

\subsection{Asymptotic geometry of the mapping class group}

Let $S$ be a compact topological surface (possibly with a boundary); the complex of curves (and arcs) of $S$, denoted by $\mathcal{X}(S)$ (or simply $\mathcal{X}$ if only one surface is involved) is the graph whose vertices are the homotopy classes of simple closed loops on $S$ which are non parallel to the boundary and arcs from the boundary to the boundary which are not homotopic to the boundary and whose edges are pairs of curves that can be made disjoint by a homotopy.

A fundamental result due to $\mathrm{H}$. Masur and $\mathrm{Y}$. Minsky asserts that $\mathcal{X}$ is hyperbolic [Ma-Mi]; this statement has been improved by B. Bowditch [Bo] who proved that the action of $M(S)$ on $\mathcal{X}$ is acylindrical. Therefore, in principle, the results in paragraph 3 can be applied to this example. However, in order to 
study monodromy groups we will need a deeper result and consider sequences in $\operatorname{Hom}(\Gamma, M(S)) /$ conj which are infinite but whose energy when acting in $\mathcal{X}$ does not diverge. The reason is that the action of $M(S)$ on $\mathcal{X}$ is not proper. The work of M. Bestvina, M. Bromberg, and K. Fujiwara [Be-Br-Fu] gives a new geometric structure on the mapping class group which enables to apply the results of Paragraph 3, and in particular Theorem 6 .

Theorem 7 (Be-Br-Fu) The mapping class group $M(S)$ of a compact surface $S$ contains a subgroup of finite index $M_{1}(S)$ which admits a product action on a finite product of hyperbolic spaces $\Pi_{i \in I} \mathcal{X}_{i}$. Furthermore the orbit map $M_{1}(S) \rightarrow \Pi_{i \in I} \mathcal{X}_{i}$ is a quasi-isometric embedding.

The spaces $\mathcal{X}_{i}$ constructed by $[\mathrm{Be}-\mathrm{Br}-\mathrm{Fu}]$ are projection complexes of complexes of curves. In order to apply the factorization Theorem 6 of Paragraph 3 we have to check that these actions are weakly acylindrical. In fact, the metric spaces $\mathcal{X}_{i}$ are defined as $C_{K}\left(\boldsymbol{Y}_{i}\right)$, where $\boldsymbol{Y}_{i}$ is a family of connected subsurfaces of $S$, and the metric space attached to a subsurface $Y$ is the complex of curves and $\operatorname{arcs} C(Y)$ of $Y$. As Bowditch proved that the action of the mapping class group of $Y$ on $C(Y)$ is acylindrical [Bo], it is enough (due to Proposition 7) to prove that the $\nu$ invariant for the action of $M(S)$ on the set $\boldsymbol{Y}$ of isotopy classes of connected subsurfaces of $S$ if finite.

Lemma 4 The action of the mapping class group on the set of isotopy classes of connected subsurfaces of $S$ has a finite $\nu$-invariant. In particular the action of $M(S)$ on $\mathcal{X}_{i}$ is weakly acylindrical.

Proof Let $g, h$ be two elements in $M(S)$ such that $h$ fixes the non empty connected surfaces $Y_{0}, g^{-1} Y_{0}, g^{-2} Y_{0} \ldots, g^{-k} Y_{0}$. Let us consider $Y_{1}, \ldots, Y_{c}$ the pieces of the Nielsen decomposition of $S$ associated with $h$ (the orbits under $h$ of connected components of the Nielsen decomposition) and $I \subset\{1, \ldots c\}$ the components on which $h$ is homotopic to the identity. For every $i$, the surface $g^{-i} Y_{0}$ is contained in a unique $Y_{\alpha}$, say in $Y_{f(i)}$. As $h$ fixes $g^{-i} Y_{0}$, this subsurface $Y_{f(i)}$, must be connected. Then either the restriction of $h$ to $Y_{f(i)}$ is the identity $(f(i) \in I)$, or it is a pseudo-Anosov and in this case, $g^{-k} Y_{0}=Y_{c}$ is fixed by $h$. Let us assume that $k>c$. Then either for two different indices $i, j \leqslant k$, $g^{-i} Y_{0}=g^{-j} Y_{0}=Y_{c}$ and $g^{j-i} Y_{0}=Y_{0}$, and $h$ fixes all $g^{k} Y_{0}$, or if $k$ is sufficiently large (greater than $\Sigma_{1 \leqslant i \leqslant c}-\xi\left(Y_{i}\right)+1$, where $\xi$ the Euler characteristic), $g^{-k} Y_{0}$ must be contained in $\cup_{0 \leqslant i<k, f(i) \in I} g^{-i} Y_{0}$. Therefore $g^{-(k+1)} Y_{0}$ is contained in a set on which $h$ induces the identity.

\subsection{Infinite sequences of homomorphisms.}

We will apply Paragraph 3.2 to create actions on $\mathrm{R}$ - trees from an infinite sequence of pairwise non conjugate homomorphisms of a finitely generated group $(\Gamma, \Sigma)$ to the mapping class group $M(S)$. At this point one could directly apply 
the work of J. Behrstock, C. Drutu and M. Sapir [Be-Dr-Sa] Corollary 6 from the appendix. In particular, we know that, given a group $\Gamma$ and a sequence of pairwise non conjugate homomorphisms $\varphi_{n}: \Gamma \rightarrow M(S)$ we can construct a non elliptic action of $\Gamma$ on some limit tree.

Assume furthermore that $\varphi_{n}(\Gamma)$ is not reducible. Using the weak acylindricity of the spaces $\mathcal{X}_{i}$ constructed by Bestvina, Bromberg, Fujiwara, together with Proposition 6, we will deduce (Proposition 8) that the action on the limit tree is not elementary, and that if $N$ is a finitely generated normal subgroup of the kernel of this limit action $\varphi_{n}(N)$ is finite for almost every $n$.

Let us recall very briefly the definition of this family of spaces. If $S$ is a surface, $\mathbf{Y}$ denote the set of isotopy classes of incompressible subsurfaces of $S$ which are not spheres with three holes. It is shown [Be-Br-Fu], prop. 4.8 that $\mathbf{Y}$ can be partitioned in a finite set $\mathbf{Y}=\cup_{i \in I} \mathbf{Y}_{i}$ such that the boundaries two subsurfaces in the same components always meet. Further, the partition is natural, i.e. the mapping class group contains a subgroup of finite index $M_{1}(S)$ which acts while preserving its components (the $\mathbf{Y}_{i}$ ). Recall that each $\mathbf{Y}_{i}$ is a endowed with a structure of a projection complex of metric spaces, where the metric space attached to a sub-surface is the complex of curves and arcs of this subsurface (a hyperbolic space due to the fundamental work of Masur and Minsky). The space $\mathcal{X}_{i}$ is the metric space $C_{K}\left(\mathbf{Y}_{i}\right)$, where $K$ is large enough.

In order to apply our Theorem 6 to the action of $M_{1}(S)$ on $\mathcal{X}_{i}$, we recall a fundamental result of Ivanov [Iv] concerning the mapping class group: a finitely generated subgroup of $M(S)$ which is not reducible contains a pseudo-Anosov element. Here, reducible means that the subgroup permutes a finite set of disjoint simple closed curves, up to homotopy, equivalently is elliptic while acting in the complex of curves of $S$.

Lemma 5 Let $G \subset M(S)$ be a f.g. irreducible group and $N \subset G$ a finitely generated normal subgroup. Then $N$ contains a pseudo-Anosov.

If an elementary subgroup of $M_{1}(S)$ acting on $\mathcal{X}_{i}$ contains a pseudo Anosov, it is virtually cyclic.

Proof If a $N$ contains no pseudo-Anosov and is finitely generated, then its action on the complex of curves is elliptic and the set of curves preserved by $N$ is bounded (for instance because of the acylindricity of the action). Applying Ivanov's Theorem, we get that $G$ must be reducible.

As the vertices of the projection complex associated to $\mathbf{Y}_{i}$ are sub-surfaces, Lemma 2 in 4.2 implies that a pseudo-Anosov $\psi$ cannot act as an elliptic or parabolic isometry of $P_{K}\left(\mathbf{Y}_{i}\right)=\mathcal{X}_{i}$. The elementary subgroup generated by $\psi$ acts by translation on its stable axis, with kernel a subgroup $K$ which is reducible as it fixes the stable vertices of this stable axis. If it is not finite, the set of curves it fixes in the complex of curve is bounded (by acylindricity) and cannot be $\psi$ invariant. 
Now, let $\Gamma$ be a group and $\varphi_{n}: \Gamma \rightarrow M(S)$ be an infinite sequence of pairwise non conjugate homomorphisms.

Let $\Gamma_{1} \subset \Gamma$ is the intersection of all kernel of all homomorphisms of $\Gamma$ to the permutation group of the set $I$. As $\Gamma$ is finitely generated and $I$ is finite, the subgroup $\Gamma_{1}$ is of finite index in $\Gamma$, and for every $n, \varphi_{n}\left(\Gamma_{1}\right) \subset M_{1}(S)$. Thus, the restriction of $h_{n}$ to $\Gamma_{1}$ does not permute the factors $\mathcal{X}_{i}$.

Let us fix a generating system $\Sigma$ of $\Gamma_{1}$. Simultaneously, we fix a generating system of $M(S)$ and denote by $|\gamma|$ the word length of an element $\gamma \in M(S)$.

If $\varphi: \Gamma_{1} \rightarrow M(S)$ is an homomorphism, the energy of $\Gamma_{1}$ acting on the Cayley graph of $M(S)$ is $E(\varphi)=\operatorname{Min}_{\gamma \in M(S)} \operatorname{Max}_{s \in \Sigma}\left|\gamma^{-1} \varphi(s) \gamma\right|$. As they are only a finite number of elements of a given length, there are only a finite number of conjugacy classes of homomorphisms whose restriction to $\Gamma_{1}$ have a given energy.

In our case, the homomorphisms $\left(\varphi_{n}\right)_{n \in \mathbb{N}}$ are pairwise non conjugate, therefore $\lim _{n \rightarrow \infty} E\left(\varphi_{n}\right)=\infty$.

For each $n$ choose $\gamma_{n}$ such that the minimum is achieved, and let us replace $\varphi_{n}$ by its conjugate $\gamma_{n} \cdot \varphi_{n} \gamma_{n}^{-1}$. Let $C$ be the asymptotic cone of the mapping class group associated to the sequence $E_{n}$, namely $C=\lim _{\omega}\left(\frac{1}{E_{n}} \mathrm{Ca}(M(S)), 1\right)$.

Let us fix an origin $\alpha=\left(\alpha_{i}\right)_{i \in I}$ in $\Pi_{i \in I} \mathcal{X}_{i}$. Recall (Theorem 7) that the orbit map $M_{1}(S) \rightarrow \Pi_{i \in I} \mathcal{X}_{i}$ defined by $\phi(g)=g . \alpha=\left(g \cdot \alpha_{i}\right)_{i \in I}$ is a quasiisometric embedding, i.e there exists constants $K, L$ such that $K^{-1}-L \leq|g| \leq$ $\operatorname{Max}_{i \in I} d\left(g . \alpha_{i}, \alpha_{i}\right) \leq K .|g|$

Therefore, if $n$ is fixed and $e_{i, n}$ is the energy of the action $\varphi_{n}$ of $\Gamma_{1}$ on $X_{i}$ computed at the point $\alpha_{i} K^{-1} E\left(\varphi_{n}\right)-L \leq \operatorname{Max}_{i \in I} e_{i, n} \leq K E\left(\varphi_{n}\right)$.

Fort each $n$, let $i_{n}$ be an index for which this maximum is achieved. As the sequence $\left(i_{n}\right)$ takes only a finite number of values, if $j=\lim _{\omega} i_{n}$, for $\omega$-almost all integer $n, i_{n}=j$.

If $i$ is another index, then for $\omega$-almost all integer $n, 0 \leq \frac{e_{j, n}}{e_{i, n}} \leq 1$, and either $\lim _{\omega} \frac{e_{j, n}}{e_{i, n}}=0$ or $\lim _{\omega} \frac{e_{j, n}}{e_{i, n}}=\lambda_{j}>0$.

Now, let $T_{i}$ be the minimal invariant subtree of the limit tree of the sequence of hyperbolic spaces $\lim _{\omega} \frac{1}{E\left(\varphi_{n}\right)}\left(X_{i}, \alpha_{i}\right)$. Note that, if $\lim _{\omega} \frac{e_{j, n}}{e_{i, n}}=0$, the origin is fixed by all the generators, and $T_{i}$ is reduced to a point. But if $\lim _{\omega} \frac{e_{j, n}}{e_{i, n}}=$ $\lambda_{j}>0$, the limit tree $T_{i}$ is, up to the factor $\lambda_{i}$, the same tree as the minimal subtree of the limit tree $\lim _{\omega}\left(\frac{1}{e_{i, n}} \mathcal{X}_{i}, \alpha_{i}\right)$ defined by the sequence of actions of $\Gamma_{1}$ on $\mathcal{X}_{i}$.

Proposition 8 Assume that for all $n$, the group $\varphi_{n}\left(\Gamma_{1}\right)$ is neither reducible nor virtually abelian. Then, $\Gamma_{1}$ admits a non elementary action on a tree, more precisely, for some $i$, the action of $\Gamma_{1}$ on $T_{i}$ is not elementary.

Let $N$ be a f.g. normal subgroup contained in the kernel of the action of $\Gamma_{1}$ 
on this tree $T_{i}$. Then for infinitely many $n, \varphi_{n}(N)$ is finite. In particular, the restriction of $\varphi_{n}$ to a subgroup of finite index in $\Gamma_{1}$ factors through $\Gamma_{1} / N$.

\section{Proof}

Let $O \subset C$ the orbit of the natural base point $\lim _{\omega} e$ for the limit action of $\Gamma_{1}$. Dividing the distances by $E\left(\varphi_{n}\right)$ we see that the orbit map induces a bi-lipshitz embedding $\phi: O \subset C \rightarrow \prod_{i \in I^{\prime}} \lim _{\omega}\left(\frac{1}{e_{i, n}} \mathcal{X}_{i}, \alpha_{i}\right)$, where $I^{\prime} \subset I$ is the subset for which $\lambda_{i}>0$. This embedding is equivariant for limit the action of $\Gamma_{1}$ on these spaces.

The fact that for some $i$, the action of $\Gamma$ on $T_{i}$ is not elliptic, follows from [Be-Dr-Sa], Section 6, Theorem 6.2, who proved that the orbit $O$ of the group $\Gamma_{1}$ on $C$ is unbounded. As the embedding of $C$ in the product of the limit spaces is bi-Lipshitz (hence metrically proper) it cannot project onto bounded orbits. For some index $i$ the action of $\Gamma_{1}$ on the limit tree is unbounded, and $\Gamma_{1}$ is not elliptic in $T_{i}$.

If $T_{i}$ is a line (equivalently if the limit action is elementary) Proposition 6 (3) proves that for some $n \varphi_{n}(\Gamma)$ acting on $\mathcal{X}_{i}$ is elementary. As it contains a pseudo-Anosov, it must be virtually abelian (Lemma 5), contradiction.

For the second point, note that, due the weak acylindricity property (Proposition $6,(2))$, the group $\varphi_{n}(N)$ is elementary elliptic, hence reducible. Due to Lemma 5 it must be finite.

\subsection{Holomorphic family of Riemann surfaces}

Let $X$ be a compact Kähler manifold. A holomorphic family of Riemann surfaces of genus $g$ over $X$ is a pair $(Z, \pi)$ where $Z$ is a compact complex manifold and $\pi: Z \rightarrow X$ a holomorphic fibration such that the fibers of $\pi$ are Riemann surfaces of genus $g$.

Let $x_{0}$ be a base point in $X, Z_{x_{0}}=\pi^{-1}\left(x_{0}\right)$ its fiber and $S$ the underlying topological surface. Let $\mathcal{T}=\mathcal{T}_{g}$ be the Teichmüller space of $S$. Recall that $\mathcal{T}$ is defined as the set of isotopy classes of complex structures on $S$, i.e. the quotient of the set of complex structures on $S$ by the group of diffeomorphisms of $S$ that are isotopic to the identity. The mapping class group $M(S)$, being defined as the group of isotopy classes of diffeomorphisms of $S$, naturally acts on $\mathcal{T}$.

As the bundle $Z \rightarrow X$ is locally trivial, it determines an homomorphism $\varphi: \pi_{1}\left(X, x_{0}\right) \rightarrow M(S)$ called the monodromy.

Simultaneously the Teichmüller theory enables to construct a complex structure on $\mathcal{T}$ and an holomorphic map $\Phi$ from the universal cover $\left(\tilde{X}, x_{0}\right)$ of $X$ to $\mathcal{T}$, such that $\Phi(p)$ is the isotopy class of the complex structure on the image of $p$ in $X$.

Let us briefly recall the construction of this map $\Phi$, called the classifying map. As we will use it in the slightly more general context of orbifolds, we 
present this construction in terms of homomorphisms of étale groupoïds rather than analytic continuation of holomorphic maps.

A differentiable trivialization $\Psi: U \times S \rightarrow Z$ over a connected open neighborhood $U$ of $x_{0}$ gives a map $\Phi_{U}$ sending $x$ to the complex structure on $S$ obtained by pulling back by $\Psi(x,):. S \rightarrow Z$ the complex structure of $Z$. A fundamental result (due to Teichmüller and Bers) endows $\mathcal{T}$ with a structure of a complex manifold so that the map $\Phi_{U}$ is holomorphic (see for instance the beautiful text of Weil [We]). One proves that this map is uniquely defined (i.e it does not depend on the trivialization over $U$ ) modulo the action of the finite group of complex automorphism of the Riemann surface $\operatorname{Aut}\left(S_{x_{0}}\right)$.

Let $\left(\tilde{X}, x_{0}\right)$ be the universal cover of $X$, computed at the base point $x_{0}$.

Let $\left(U_{\alpha}\right)_{\alpha \in A}$ be a covering of $X$ by open sets which is an atlas for the complex structure and over which the family has a trivialization. We may assume that the intersections $U_{\alpha} \cap U_{\beta}$ are empty or contractible. Let us consider holomorphic maps $\Phi_{\alpha}: U_{\alpha} \rightarrow \mathcal{T}$ over each of these sets such that the complex structure over a point $p$ is isomorphic to $\Phi_{\alpha}(p)$. The family $\left(\Phi_{\alpha}\right)$ is well defined up to the action of $M\left(S_{0}\right)$. For every non empty intersection $U_{\alpha} \cap U_{\beta}$ there exists a mapping class $g_{\alpha, \beta}$ such that $\Phi_{\alpha}=g_{\alpha, \beta} \Phi_{\beta}$. In the langage of étale groupoïds ([Br-Ha] Chapter III.G.2), the set of map $\left(\Phi_{\alpha}\right)_{\alpha \in A}$ and isometries $g_{\alpha, \beta}$ is a continuous homomorphism from the étale groupoïd of holomorphic changes of charts defining $X$ to the (mapping class) group of isometries of the Teichmüller space endowed with its Weil-Peterson Kähler structure. From the developing Lemma ([Br-Ha] Proposition 3.17, p. 611), one deduces that there exists a unique holomorphic map $\Phi$ from the universal cover of $X$ computed at the point $x_{0}$ to the Teichmüller space which extends the map $\Phi_{0}$ defined on $U_{o} \ni x_{0}$. Moreover this map is equivariant for the monodromy $\varphi: \pi_{1}\left(X, x_{0}\right) \rightarrow M(S)$.

This (rather abstract) point of view allows us to immediately extend the definitions to the case where $X$ is a complex orbifold, viewed as an étale groupoïd (see $[\mathrm{Br}-\mathrm{Ha}]$ ). If the orbifold is developable, a non isotrivial family of Riemann surfaces over $X$ is nothing else but a family $\tilde{\pi}: \tilde{Z} \rightarrow \tilde{X}$ over the universal cover of $X$, endowed with a $\tilde{\pi}$-equivariant action of the fundamental group $\pi_{1}^{\text {orb }}(X)$.

An important case is the one where the base is a hyperbolic (hence complex) 2-orbifold. Such an orbifold is developable, its fundamental group is a lattice in PSL $2(\mathrm{R})=\operatorname{Aut}(U)$ the group of automorphisms of the unit disc. Conversely, let $\Gamma \subset \operatorname{PSL}(2, \mathrm{R})$ be a Fuchsian group, then $X=U / \Gamma$ is a complex 2-orbifold. A family of Riemann surfaces over $X$ is gives a representation $\varphi: \Gamma \rightarrow M(S)$ and a holomorphic $\Gamma$-equivariant map from $U \rightarrow \mathcal{T}$.

Summing up, a holomorphic family $\pi: Z \rightarrow X$ of Riemann surfaces of genus $g$ over a complex manifold (orbifold) $X$ determines two objects:

1. The monodromy $\varphi$, which is a homomorphism from the (orbifold) fundamental group of $X$ to the mapping class group of its fiber.

2 . The classifying map which is an holomorphic $\varphi$-equivariant map from the universal cover of $X$ to the Teichmüller space of $S$. 
One says that the family is isotrivial if $\Phi$ is constant (equivalently, the image of $\varphi$ is finite).

Definition 8 Let $(Z, \pi, X)$ be a family of Riemann surfaces over $X$. One says that $\pi$ factors through a curve if there exists a hyperbolic 2-orbifold $Y$, a holomorphic map with connected fibers $F: X \rightarrow Y$ and a family $\left(Z^{\prime}, \pi^{\prime}, Y\right)$ over $Y$ such that $Z=F^{*} Z^{\prime}$.

Let us recall that the Teichmüller space is contractible (it is homeomorphic to a ball) and let $\Omega_{\mathrm{WP}}$ be the imaginary part of the Weil-Peterson Kähler metric (defined in [We]). As $\mathcal{T}$ is contractible and the action of $M(S)$ is properly discontinuous with finite stabilizers, this space serves as a classifying space of $M(S)$ over $\mathrm{R}$, and the form $\Omega_{\mathrm{WP}}$ determines a cohomology class in the mapping class group $\left[\Omega_{\mathrm{WP}}\right] \in H^{*}(M(S), \mathrm{R})$, the Weil-Peterson class.

Proposition 9 The following assertions are equivalent:

i. The family factors through a curve

ii. $\varphi^{*}\left(\left[\Omega_{\mathrm{WP}}\right]\right)^{2}=0\left(\right.$ in $\left.H^{4}(\Gamma, \mathrm{R})\right)$

iii. The complex rank of the (holomorphic) classifying map $\Phi$ is 1 .

Proof The implications $i \Rightarrow i i$ and $i i i \Rightarrow i$ are obvious. Assume that the complex rank of $\Phi$ is $r \geqslant 2$. As $\Phi$ is holomorphic and $\Omega_{\mathrm{WP}}$ is a Kähler form on the Teichmüller space, $\Phi^{*}\left(\Omega_{\mathrm{WP}}^{r}\right)$ is a non zero harmonic $(r, r)$-form on $\tilde{X}$, which is $\Gamma$-equivariant and defines a non zero harmonic form of degree $2 r$ on $X$, providing a non zero class in $H^{2 r}(X, \mathrm{R})$. As the Teichmüller space is contractible, this class vanishes on $\pi_{k}(X)$ for every $k \in\{1, \ldots, 2 r\}$ and belongs to the image of the cohomology of the fundamental group by the natural map $H^{*}(\Gamma, \mathrm{R}) \rightarrow H^{*}(X, \mathrm{R})$.

Corollary 1 The fact that a family of Riemann surfaces over a manifold $X$ factors only depends on its monodromy (the morphism $\varphi: \Gamma=\pi_{1}(X) \rightarrow M(S)$ ), not on the manifold.

If there exists a finite cover $X_{1}$ of $X$ such that the pullback of the bundle $Z$ factors through a curve, then $Z$ itself factors.

Proof. For the second point, note that the family factor if and only $\mathrm{f}$ $\varphi^{*}\left(\omega_{W P}\right)^{2}=0$, and the map $H^{2,2}(X) \rightarrow H^{2,2}\left(X_{1}\right)$ is injective, as $X$ is a Kähler manifold. The following Theorem, due to Imayoshi and Shiga [Im-Sh] (see also $[\mathrm{McM}])$ is a key point in their proof of Parshin's finiteness Theorem.

Theorem 8 Let $\varphi$ be the monodromy of a family of Riemann surfaces; then the image of $\varphi$ cannot be reducible or virtually cyclic. 
Proof The first point is just a reformulation of Paragraph 4, Case 2 from [Im-Sh], or the "Irreducibility" Part in the proof of Theorem 3.1, page 126 in $[\mathrm{McM}]$. In order to check that the image cannot be virtually $\mathrm{Z}$, note that by contradiction this would imply that $\varphi^{*} \Omega=0$, as $H^{2}(\mathrm{Z}, \mathrm{R})=0$. Thus $\Phi$ would be constant.

\subsection{Finiteness of monodromies.}

Let us say that a morphism $\Gamma \rightarrow M(S)$ from a Kähler group to the mapping class group of a topological surface $S$ of genus at least 2 is a monodromy if it can be realized as the monodromy of a family of Riemann surfaces over a Kähler manifold whose fundamental group is $\Gamma$.

Theorem 9 Let $\Gamma$ be a Kähler group. Then there are only finitely many conjugacy classes of monodromies $\varphi: \Gamma \rightarrow M$ which do not factor through a Riemann surface.

Proof Let $\varphi_{n}$ be an infinite sequence of pairwise non conjugate monodromies. Theorem 8 enables to apply Proposition 8 together with the factorization Theorem (Theorem 6). We construct a finite index subgroup of $\Gamma$ which fibers over a Riemann surface group $\Lambda$ such that if $N$ is the kernel of this fibration, $\left.\varphi_{n}\right|_{N}$ is finite. Thus, $\Gamma$ admits a finite index subgroup such that $\varphi_{n}$ restricted to this subgroup factors through a Riemann surface. By Corollary $1, \varphi_{n}$ itself factors through a Riemann surface, a contradiction.

Corollary 2 The number of non isotrivial families over a compact Kähler manifold $X$ can be bounded in terms of its fundamental group.

Proof The case of a Riemann surface is a Theorem of L. Caporaso [Ca]. Applying simultaneously Proposition 2 and corollary 1, we are reduced to study families which do not factor through a curve. According to Theorem 8, we know that these families can only have finitely many possible monodromies.

In order to conclude, we need to prove the following Lemma, which extends the Rigidity Theorem of Imayoshi and Shiga [Im-Sh], page 212,see also [McM].

Lemma 6 On a given compact Kähler manifold, a non isotrivial family is determined by its monodromy.

Proof The case of curves is exactly the Rigidity Theorem of Imayoshi and Shiga. The case where $X$ is a projective manifold follows by induction on the dimension, while considering the restriction of the family to hyperplane sections. For the case of a Kähler manifold, let us first assume that the monodromy group $\varphi(\Gamma)$ of a fixed family $Y \rightarrow X$ do not contain element of finite order.

Consider the algebraic reduction $V$ of $X$ (see [Ue], p. 24-25). There exists another Kähler manifold $X^{*}$ bimeromorphically equivalent to $X$ via a meromorphic map $F$ (which induces an isomorphism on fundamental groups), a 
projective manifold $V$ and a holomorphic map $r: X^{*} \rightarrow V$ such that for every holomorphic map to $X \rightarrow \mathbb{C} P^{n}$, there exists an holomorphic map $g: V \rightarrow \mathbb{C} P^{n}$ such that $f=g o F$.

If $\Phi: X \rightarrow M_{g}$ is the classifying map of our family, as $M_{g}$ is a quasiprojective manifold, every fiber of the algebraic reduction $V$ are send to point in $M_{g}$, the monodromy factors through $r_{*}$, and, as the monodromy group does not contain elements of finite order, the family over $X$ is the pullback of a family over $V$. Therefore it is determined by its monodromy.

Let us now consider the general case. Let $M^{\prime}(S)$ a torsion free subgroup of finite index in $M(S)$. If $\varphi: \pi_{1}(X) \rightarrow M(S)$ is given, we can consider the étale cover $X^{\prime}$ of $X$ associated to the subgroup $\varphi^{-1}\left(M^{\prime}(S)\right)$. As a family over $X$ is determined by its pullback on $X^{\prime}$, we see that a family over $X$ is determined by the restriction of $\varphi$ to this subgroup of finite index.

\section{Bibliography}

[ABCKT] Amoros, J. Burger, M., Corlette, K., Kotschick, D. Toledo, D. Fundamental groups of compact Kähler manifolds, Mathematical Surveys and Monographs, 44, AMS Providence RI, 1996

[Ar] Arakelov, S. Ju. Families of algebraic curves with fixed degeneracies. Isz. Akad. Nauk SSSR 35 (1971) 1269-1293.

[Be-Dr-Sa]. Behrstock, J. Drutu, C. Sapir, M. Median structures on asymptotic cones and homomorphisms into mapping class groups. Proc. Lond. Math. Soc. (3) 102 2011, pp 503-554 and addendum pp. 555-562.

[Be1] Bestvina, M. Degeneration of the hyperbolic space. Duke Math. J. 56 (1988) 143-146.

[Be2] Bestvina, M. R-trees in topology, geometry, and group theory. Handbook of geometric topology, 55-91. North Holland, Amsterdam. 2002.

[Be-Br-Fu] Bestvina, M. Bromberg, K. Fujiwara, K. Constructing group actions on quasi-trees and applications to mapping class groups. Pub. Math. IHES, to appear.

[Bo] Bowditch, Brian H. Tight geodesics in the curve complex. Inv. Math. 171 (2008) 281-300.

[Br-Ha] Bridson, M. Haefliger, A. Spaces of Non-Positive Curvature, Springer, Berlin-Heidelberg, 1999.

[Ca] Caporaso, L. On certain uniformity properties of curves over function fields. Composition Math. 130 (2002),1-19.

[Ca-La] Cantat, S. Lamy, S. Normal subgroups in the Cremona group. Acta. Math. 210 (2013), 31-94. 
[Ca-To]. Carlson, J. Toledo, D. Harmonic mappings of Kähler manifolds to locally symmetric spaces, Pub. Math. IHES (1989) 173-201.

[Cat] Catanese, F. Differentiable and deformation type of algebraic surfaces. Symplectic 4-manifolds and algebraic surfaces, 55-167. LNM 1938, Springer 2008.

[Co-Si] Corlette, K. Simpson, C. On the classification of rank 2 representations of quasi-projective fundamental groups. Compositio Math. 144 (2008), 1271-1331.

[Co-De-Pa] Coornaert, M. Delzant, T. Papadopoulos, A. Géométrie et théorie des groupes. Les groupes hyperboliques de Gromov. Lecture Note in Maths 1441 Springer-Verlag, Berlin, 1990

[Co] Coulon, R. Partial periodic quotient of groups acting on a hyperbolic space. Preprint arXiv:1311.0855

[De] Delzant, T. Trees, valuations and the Green-Lazarsfeld set. Geom. Funct. Anal. 18 (2008), 1236-1250.

[De2] Delzant, T. L'invariant de Bieri Neumann Strebel des groupes fondamentaux des variétés kähleriennes. Math. Ann. 348 (2010) 119-125.

[De-Gr] Delzant, T, Gromov M. Courbure mésoscopique et théorie de la toute petite simplification. J. Topol. 1 (2008), 804-836.

[Gr] Gromov, M. Hyperbolic groups. Essays in group theory, 75-263. MSRI Pub. Springer 1987.

[Gr2] Gromov, M. Asymptotic invariants of infinite groups. London Math. Soc. LNS 1982

[Gr-Sh] Gromov, M. Schoen, R. Harmonic maps into singular spaces and p-adic super rigidity for lattices in groups of rank one. Pub. IHES 76 (1992) $165-246$.

[H-M] J. Harris, I. Morrison, Moduli of Curves, Graduate Texts in Mathematics 187, Springer-Verlag, 1998.

[Im-Sh] Imayoshi, Yôichi; Shiga, Hiroshige, A finiteness Theorem for holomorphic families of Riemann surfaces. Holomorphic functions and moduli, Vol II, 207-219, MSRI Pub, Springer 1988.

[Iv] Ivanov, N.V. Subgroups of Teichmüller modular groups, American Mathematical Society, Providence, RI, 1992

[Ka] Kapovich, M. Hyperbolic Manifolds and Discrete Groups: Lectures on Thurston's Hyperbolization, Birkhauser's series "Progress in Mathematics", 2000.

[Ko-Sh] Korevaar, N. Schoen, R. Global existence Theorems for harmonic maps to non locally compacts spaces. Comm. Anal. Geom. 5 (1997), 333-387.

[Ma-Mi] Masur, H. Minsky, Y. Geometry of the complex of curves. I, II. 
Hyperbolicity. Inv. math. 138 (1999) 103-149, and Geom. Funct. Anal. 10 (2000) 902-974.

[McM] McMullen, C. From dynamics on surfaces to rational points on curves. Bull. AMS 37 (2000) 119-140.

[Par] Parshin, A.N. Algebraic curves over function fields. Dokl. Akad. Nauk SSSR 32 (1968), 1191-1219.

[Pa] Paulin, F. Topologie de Gromov équivariante, structures hyperboliques et arbres réels, Inv. Math. 94 (1988) 53-80.

[Se] Sela Z. Diophantine geometry over groups. I. Makanin-Razborov diagrams. Pub. Math. IHES 93, (2001) 31-205.

[Si] Siu, Y.T The complex-analyticity of harmonic maps and the strong rigidity of compact Khler manifolds. Ann. of Math. (2) 112 (1980), no. 1, 73111.

[Su] Sun, X. Regularity of harmonic map to trees. Amer. J. Math. 125 (2003) 737-771

[Th] Thurston, W.P. The Geometry and Topology of three-manifolds, Notes http://library.msri.org/books/gt3m/

[Ue] Ueno, K. Classification theory of algebraic varietes and compact complex spaces, LNM 439. Springer 1975.

[We] Weil, A. Modules des surfaces de Riemann, Séminaire Bourbaki, 195658 exposé 168 , p. 413-19.

[W] Wolpert, S.A. Families of Riemann surfaces and Weil-Petersson geometry. CBMS Regional Conference Series in Mathematics, 113.American Mathematical Society, Providence, RI, 2010 